SISSA $/ 44 / 92 / \mathrm{EP}$

IFUM $/ 443 / \mathrm{FT}$

\title{
ALE Manifolds and Conformal Field Theories
}

\author{
Damiano Anselmi, Marco Billó, Pietro Fré \\ SISSA - International School for Advanced Studies, via Beirut 2-4, 34140 Trieste, Italy \\ and I.N.F.N. - Sezione di Trieste - Trieste, Italy \\ Luciano Girardello \\ Dipartimento di Fisica, Università di Milano, via Celoria 6, 20133 Milano, Italy \\ and I.N.F.N. - Sezione di Milano, Milano, Italy
}

Alberto Zaffaroni

SISSA - International School for Advanced Studies, via Beirut 2-4, 34140 Trieste, Italy and I.N.F.N. - Sezione di Trieste - Trieste, Italy

\begin{abstract}
We address the problem of constructing the family of $(4,4)$ theories associated with the sigma-model on a parametrized family $\mathcal{M}_{\zeta}$ of Asymptotically Locally Euclidean (ALE) manifolds. We rely on the ADE classification of these manifolds and on their construction as HyperKähler quotients, due to Kronheimer. So doing we are able to define the family of $(4,4)$ theories corresponding to a $\mathcal{M}_{\zeta}$ family of ALE manifolds as the deformation of a solvable orbifold $\mathbf{C}^{2} / \Gamma$ conformal field-theory, $\Gamma$ being a Kleinian group. We discuss the relation among the algebraic structure underlying the topological and metric properties of self-dual 4-manifolds and the algebraic properties of non-rational $(4,4)$-theories admitting an infinite spectrum of primary fields. In particular, we identify the Hirzebruch signature $\tau$ with the dimension of the local polynomial $\operatorname{ring} \mathcal{R}=\frac{\mathbf{C}[x, y, z]}{\partial W}$ associated with the ADE singularity, with the number of non-trivial conjugacy classes in the corresponding Kleinian group and with the number of short representations of the $(4,4)$-theory minus four.
\end{abstract}

hep-th/9304135 


\section{Introduction}

Recently a considerable amount of efforts have been devoted to the construction of exact conformal field theories describing superstring propagation on non-trivial space-time geometries [1, 2, 3, 4, 5]. One research-line has focused on the construction of Blackhole-like solutions while another one aimed at the search for gravitational-instanton-like backgrounds. Following the pioneering work of [3] some of us have recently established the following result [4]:

Stringy gravitational instantons can be identified with $(4,4) c=6$ conformal fieldtheories in the very same way as $(2,2) c=9$ theories can be identified with Calabi-Yau three-folds

In particular in [4] we have introduced a generalization of the notion of HypeKähler manifolds, that incorporates also the torsion background and that defines the most general conditions under which a $(1,1)$-supersymmetric $\sigma$-model develops a $(4,4)$-global supersymmetry, leading to a $(4,4)$ superconformal field theory.

Central to the analysis of [⿴囗十 is the concept of abstract Hodge-diamond. Let us briefly review it. We start by treating the $(4,4) c=6$ theory as a $(2,2)$ one (something that we can always do) and we denote the $(2,2)$ primary fields by $\Psi\left(\begin{array}{c}h, \tilde{h} \\ q, \tilde{q}\end{array}\right)(z, \bar{z})$, where $h, \tilde{h}$ are the left (right) conformal weights and $q, \tilde{q}$ are the left (right) $U(1)$-charges. In the $(4,4)$ language the primary fields are instead denoted by $\Psi\left[\begin{array}{c}h, \tilde{h} \\ J, \tilde{J}\end{array}\right]^{m, \tilde{m}}(z, \bar{z})$ where $h, \tilde{h}$ are the left (right) conformal weights, $J, \tilde{J}$ the left (right) isospins and $m, \tilde{m}$ their third components. The $(4,4)$ representations put together the $(2,2)$ primary fields in bigger multiplets. In particular the relation between the $U(1)$ charges $q, \tilde{q}$ and the isospin third components is $q=2 m(\tilde{q}=2 \tilde{m})$. This happens because:

$$
\begin{aligned}
& \varphi(z)=\frac{1}{\sqrt{2}} \tau(z) \\
& \tilde{\varphi}(\bar{z})=\frac{1}{\sqrt{2}} \tilde{\tau}(\bar{z})
\end{aligned}
$$

where $\varphi(z)$ is the free bosonic field that bosonizes the left $U(1)$ current $j(z)=\partial \varphi(z)$ while $\tau(z)$ is the field that bosonizes the level one $S U(2)$ algebra contained in the $c=6, N=4$ algebra: $A_{3}(z)=\partial \tau(z), A^{ \pm}(z)=\exp ( \pm \tau(z))$ (similarly for the right sector). Using these notations we can attach to a $(2,2) c=6$ theory with integral $U(1)$ charges an array of numbers (the abstract Hodge-diamond)

$$
\begin{array}{ccccc} 
& \multicolumn{3}{c}{h^{(0,0)}} & \\
h^{(2,0)} & & h^{(1,1)} & h^{(0,1)} & \\
& h^{(1,2)} & & h^{(2,1)} & \\
& & h^{(2,2)} & &
\end{array}
$$

where $h^{(p, q)}$ denotes the number of chiral-chiral primary fields $\Psi\left(\begin{array}{c}p / 2, q / 2 \\ p, q\end{array}\right)(z, \bar{z}) \quad(a=$ $\left.1, \ldots, h^{(p, q)}\right)$. These fields span linear spaces that we name $\mathcal{H}^{(p, q)}$, the abstract Dolbeault 
cohomology groups. We recall that primary chiral fields are those admitting a regular operator product expansion with one of the supercurrents $\left(G^{+}\right.$in this case) and are characterized by the equation $q=2 h$ (primary antichiral fields have regular operator product with the other supercurrent $\left(G^{-}\right)$and are characterized by $q=-2 h$ ). In a unitary (not necessarily non-degenerate [6]) (2,2)-theory there is a bound on the maximal $U(1)$-charge of primary chirals: $q_{\max }=\frac{c}{3} \Rightarrow h_{\max }=\frac{c}{6}$. Actually both in the left and right sectors there is a unique top chiral primary $\rho\left(\begin{array}{c}\frac{c}{6}, 0 \\ \frac{c}{3}, 0\end{array}\right)(z, \bar{z})$ and $\tilde{\rho}\left(\begin{array}{l}0, \frac{c}{6} \\ 0, \frac{c}{3}\end{array}\right)(z, \bar{z})$. Furthermore another important general property of $(2,2)$-theories, namely the spectral flow, allows to relate different chiral primaries. In the $c=6$ case, by means of the operations

$$
\begin{aligned}
U_{(1,0)}: e^{i \sqrt{\frac{c}{3}} \varphi(z)} \Psi\left(\begin{array}{l}
\frac{p}{2}, \frac{q}{2} \\
p, q
\end{array}\right)(z, \bar{z}) ? & =\Psi\left(\begin{array}{l}
\frac{p}{2}+1, \frac{q}{2} \\
p+2, q
\end{array}\right)(z, \bar{z}) \\
U_{(0,1)}: e^{i \sqrt{\frac{c}{3}} \tilde{\varphi}(\bar{z})} \Psi\left(\begin{array}{l}
\frac{p}{2}, \frac{q}{2} \\
p, q
\end{array}\right)(z, \bar{z}) & =\Psi\left(\begin{array}{c}
\frac{p}{2}, \frac{q}{2}+1 \\
p, q+2
\end{array}\right)(z, \bar{z}) \\
U_{(1,1)}: e^{i \sqrt{\frac{c}{3}}(\varphi(z)+\tilde{\varphi}(\bar{z}))} \Psi\left(\begin{array}{l}
\frac{p}{2}, \frac{q}{2} \\
p, q
\end{array}\right)(z, \bar{z}) & =\Psi\left(\begin{array}{l}
\frac{p}{2}+1, \frac{q}{2}+1 \\
p+2, q+2
\end{array}\right)(z, \bar{z})
\end{aligned}
$$

we realize the following isomorphisms $\mathcal{H}^{(p, q)} \approx \mathcal{H}^{(p+2, q)} \approx \mathcal{H}^{(p+2, q+2)}$ that, together with the previous information on the existence of a unique top chiral primary imply the following structure for the abstract Hodge diamond of the most general $(2,2) c=6$ theory:

$\begin{array}{ccccc} & h^{(1,0)} & 1 & & \\ & h^{(1,1)} & & \\ & h^{(1,0)} & & h^{(1,0)} & \\ & & 1 & & \end{array}$

regardless whether the theory is rational (with a finite number of primaries) or not (with an infinite number of primaries as those associated with non compact target manifolds). In the $(4,4)$ language the crucial $h^{(1,1)}$ number just counts the short representations, whose lowest components are the fields $\Psi_{A}\left[\begin{array}{c}\frac{1}{2}, \frac{1}{2} \\ \frac{1}{2}, \frac{1}{2}\end{array}\right]^{m, \tilde{m}}(z, \bar{z})$, while $h^{(1,0)}$ counts the short representations $\Psi_{A}\left[\begin{array}{c}\frac{1}{2}, 0 \\ \frac{1}{2}, 0\end{array}\right]^{m}(z, \bar{z})$, if present. In the case where the target space of the $\sigma$ model is a compact torsionless manifold the abstract Hodge diamond is nothing else but the geometrical Hodge diamond, displaying the dimensions of the Dolbeault cohomology groups $H^{(p, q)}$ of harmonic (p,q)-forms. So in the case of the rational $(4,4)$-theories associated with the $K_{3}$-manifold and with the torus $T^{4}$ we have:

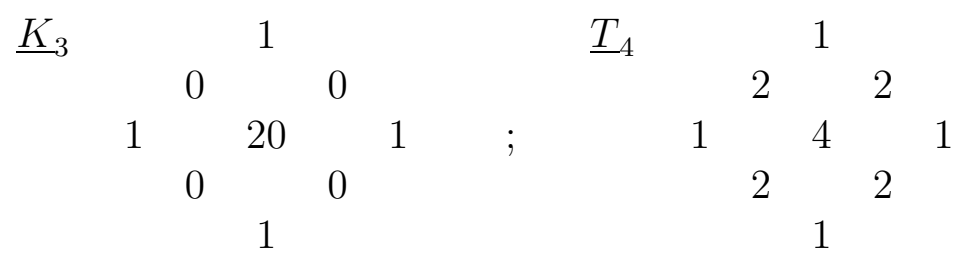


In the case where the $(4,4)$ theory under consideration corresponds to a $\sigma$-model on a non-compact HyperKähler, or generalized HyperKähler manifold, the geometrical interpretation of the abstract Hodge diamond is more involved. Yet, as we stressed in [4], it still provides the correct counting for the zero-energy excitations of the light particles moving in the instanton background. Indeed we showed how to construct the emission vertices of all such excitations in terms of representations of the abstract $(4,4)$ theory. In particular we obtained the following formulae for the zero-mode counting:

$$
\begin{aligned}
\text { \# graviton zero modes } & =3\left(h^{(1,1)}-1\right)+1 \\
\text { \# axion zero modes } & =h^{(1,1)}+2 \\
\text { \# gravitino zero modes } & =2 h^{(1,1)}+4 h^{(1,0)} .
\end{aligned}
$$

An explicit example studied in [4] is given by the $S U(2) \otimes \mathbf{R}$ instanton of [3], [7], [8], and [9], where the $(4,4)$-theory is constructed in terms of an $S U(2)$ current algebra at level $k$ and a supersymmetric Feigin-Fuchs boson. This model has an untwisted sector where the abstract Hodge-diamond is the same as the abstract Hodge diamond of the $T^{4}$-torus recalled in eq. 5. Shortly after [4] Kounnas et al [5] have shown that the same model has also a twisted sector containing additional short-representations, so that the abstract Hodge-diamond has a richer structure that will be shortly discussed in section 7 .

In the present paper we want to deepen our understanding of the algebraic structure of $(4,4)$ theories in the non compact case, which is the one relevant to gravitational instanton physics. From the geometrical point of view, the essential novelty, with respect to the compact case, is the presence of a boundary at infinity that forces upon us the distinction between absolute $H^{(p, q)}(\mathcal{M})$ and relative $H^{(p, q)}(\mathcal{M}, \partial \mathcal{M})$ cohomology groups. We have therefore to distinguish among the harmonic $(p, q)$-forms those that have compact support and those that are not normalizable. Only the first type of forms correspond to true deformations of the underlying HyperKähler (or generalized HyperKähler) structure, although all of them are associated with emission vertices of zero-energy states. Indeed we just retrieve in a different set up the usual distinction between the discrete and continuous part of the spectrum. The abstract Hodge-diamond of the $(4,4)$ theory encodes the dimensions of the absolute cohomology groups; however, one can also write the corresponding relative Hodge-diamond, listing the number of short representations that correspond to normalizable states and hence to true moduli of the superconformal theory.

To illustrate these ideas we investigate the problem of constructing the $(4,4)$-theories corresponding to the Asymptotically Locally Euclidean (ALE) self-dual four-manifolds. These gravitational instantons were extensively studied in the late seventies by physicists and mathematicians 10, 11, 12, 13, 14 and their classification, already conjectured at that time, has been finally proved in recent years by Kronheimer [15, 16], who has also provided an algorithm for their direct construction in terms of HyperKähler quotients. The algorithm in question, reviewed in the present paper in a language accessible to 
physicists, relies heavily on the point of view that regards an ALE manifold as the minimal resolution of singularities of an orbifold $\mathbf{C}^{2} / \Gamma$, where $\mathbf{C}^{2} \approx \mathbf{R}^{4}$ is the usual Euclidean flat space and $\Gamma$ is a finite Kleinian subgroup of $S U(2)$. This viewpoint, that traces back the properties of an ALE manifold to the structure of its boundary at infinity (a three sphere modded out by the Kleinian group $\Gamma$ ) is of great value in relation with conformal field theories. Indeed, precisely as it happens for the case of the compactified internal dimensions, a superstring propagates nicely on a singular orbifold variety, the corresponding conformal field theory being solvable and completely determined [17]. Hence, just as orbifold theories (see [18] for a review) have been a nice substitute for the $(2,2)$ conformal field theories of smooth Calabi-Yau manifolds, in the same way, we retrieve all the essential properties of the $(4,4)$ theories of ALE manifolds, by studying the $\mathbf{C}^{2} / \Gamma$ orbifold theory. The key difference with the compact case is that now we have a point-group but no space-group, so that there are twisted sectors but no lattice quantization of the momenta. The twist fields are the essential ingredients in the construction of the short representations, so that, at the level of the $(4,4)$ theory, the identification of the number of moduli deformations with the number of conjugacy classes of the corresponding Kleinian group becomes particularly natural.

Geometrically the ALE manifolds can be described as affine complex varieties in $\mathbf{C}^{3}$, namely the zero loci of certain polynomial constraints

$$
\{x, y, z\} \in \mathcal{M}_{\Gamma}\left(t_{1}, \ldots, t_{r}\right) \longrightarrow \tilde{W}_{\Gamma}\left(x, y, z, ; t_{1}, \ldots, t_{r}\right)=0,
$$

that are determined by the algebraic structure of the Kleinian group $\Gamma$ and that depend on $r$ complex parameters $\left\{t_{i}\right\}(i=1, \ldots, r), r$ being the number of non-trivial conjugacy classes in $\Gamma$. In the limit $t_{i}=0$ the locus (7) reduces to the orbifold $\mathbf{C}^{2} / \Gamma$ and for these values of the moduli the $(4,4)$-theory is known and solvable. We have not found, so far, any algorithm to construct the $(4,4)$ theory corresponding to a generic point in moduli-space and probably such an algorithm does not exist. Our station with ALE manifolds is therefore the same as with Calabi-Yau manifolds: we know the general properties of the associated $(4,4) \mathrm{CFT}$ (respectively $(2,2)$ theory) and we can solve it in some special point of moduli space (the orbifold limit for ALE manifolds, the orbifold limit or the Gepner tensor product point for Calabi-Yau n-folds). Generic points can be reached perturbatively by deforming around the solvable point. In the ALE case this perturbation has a particular nice geometrical interpretation: all the moduli are twisted and they are the parameters associated with the resolution of singularities.

The whole point of our paper is to show how the algebraic structure underlying the topological and metric properties of self-dual 4-manifolds (an instance of non-compact Calabi-Yau spaces) is naturally matched with the algebraic properties of non-rational $(4,4)$-theories admitting an infinite spectrum of primary fields. In [4] we have formulated the general scheme for the abstract description of a gravitational instanton as a leftright symmetric $\mathrm{N}=4$ theory and we have considered the example of the generalized HyperKähler, non asymptotically flat $S U(2) \otimes \mathbf{R}$ instanton. As remarked there, this instanton is a point in a moduli space whose other points correspond to so far unknown 
manifolds. In the present publication we compare the general superconformal framework with the case of the conventional HyperKähler ALE instantons whose moduli space is fully understood in geometrical terms.

Our paper is organized as follows. In section 2 we review the definition of ALE manifolds, discuss their cohomology and point out their classification in terms of the Kleinian groups acting on their boundary. The essential algebraic information about Kleinian groups are collected in section 3. Next, relying on the structure of the above groups, we summarize the counting of parameters for ALE metrics. Section 1 recalls the main ideas of HyperKähler quotients and explains in a language accessible to physicists the main steps of Kronheimer construction, emphasizing its relevance for the errand of the conformal field theory derivation. In particular we obtain the precise mapping between the parameters of the HyperKähler quotient utilized by Kronheimer and the deformation parameters $t_{i}$ of the potential $\tilde{W}\left(x, y, z ; t_{i}\right)$ appearing in eq.(17). Then we turn to the construction of the $\mathbf{C}^{2} / \Gamma$ orbifold conformal field-theory. This is described in section 5 where the structure of the $\mathrm{N}=4$ representations is also discussed, retrieving both the short representations associated with compact support cohomology and those associated with non-normalizable states. In section 6 we study the structure of the partition function and we analyse it in characters of the $\mathrm{N}=4$ theory. Finally in section 7 we make some concluding remarks comparing the case we have considered with the complete structure of the $S U(2) \otimes \mathbf{R}$ instanton as it appears after the new results obtained by Kounnas et al. [5], and pointing out the open problems in the interpretation of the chiral ring $\mathbf{C}[x, y, z] / \partial W$ multiplicative structure in the context of CFT.

\section{Generalities on ALE manifolds}

Non-compact HyperKähler four-manifolds

On a four-dimensional HyperKähler manifold $\mathcal{M}$ there exist three covariantly constant complex structures $\mathcal{J}^{i}: T \mathcal{M} \rightarrow T \mathcal{M}, i=1,2,3$. The metric is hermitean with respect to all of them and they satisfy the quaternionic algebra: $\mathcal{J}^{i} \mathcal{J}^{j}=-\delta^{i j}+\varepsilon^{i j k} \mathcal{J}^{k}$. In a vierbein basis $\left\{V^{a}\right\}$, the matrices $\mathcal{J}_{a b}^{i}$ are antisymmetric (by hermiticity). By covariant constancy, the three HyperKähler two-forms $\Omega^{i}=\mathcal{J}_{a b}^{i} V^{a} \wedge V^{b}$ are closed: $d \Omega^{i}=0$. Because of the quaternionic algebra constraint, the $\mathcal{J}_{a b}^{i}$ can only be either selfdual or antiselfdual; we take them to be antiselfdual: $\mathcal{J}_{a b}^{i}=-\frac{1}{2} \epsilon_{a b c d} \mathcal{J}_{c d}^{i}$. Then the integrability condition for the covariant constancy of $\mathcal{J}^{i}$ forces the curvature two-form $R^{a b}$ to be selfdual (thus automatically solving the vacuum Einstein equations).

A HyperKähler manifold is in particular a Kähler manifold with respect to each of its complex structures. Choose one of the structures (say $\mathcal{J}^{3}$ ) and fix a frame on $\mathcal{M}$ well-adapted to it. Consider then the Dolbeaut cohomology groups $H^{p, q}(\mathcal{M})$, of dimensions $h^{p, q}$. Since $\mathcal{M}$ is Ricci-flat, its first Chern class vanishes: $c_{1}(\mathcal{M})=0 ; \mathcal{M}$ is a (non-compact) Calabi-Yau manifold and therefore $h^{2,0}=h^{0,2}=1$. It is easy to see that $\Omega^{ \pm}=\Omega^{1} \pm i \Omega^{2}$ are holomorphic (resp. antiholomorphic) so that $\left[\Omega^{+}\right] \equiv H^{2,0}(\mathcal{M})$, 
$\left[\Omega^{-}\right] \equiv H^{0,2}(\mathcal{M})$, where by $\left[\Omega^{ \pm}\right]$we mean the cohomology classes of $\Omega^{ \pm} . \Omega^{3}$ is the Kähler form on $\mathcal{M}$ and $\left[\Omega^{3}\right]$ is just one of the elements of $H^{1,1}(\mathcal{M})$.

On a non-compact manifold it is worth considering the "compact-support" cohomology groups, that coincide with the relative cohomology groups of forms vanishing on the boundary at infinity of the manifold:

$$
H_{\mathbf{c}}^{p}=\frac{\left\{\mathbf{L}^{2} \text { integrable, closed } p-\text { forms }\right\}}{\left\{\mathbf{L}^{2} \text { integrable, exact } p-\text { forms }\right\}}=H^{p}(\mathcal{M}, \partial \mathcal{M}),
$$

of dimensions $b_{\mathbf{c}}^{p}$. Analogously we will consider the compact support Dolbeaut cohomology groups $H_{\mathbf{c}}^{p, q}$, of dimensions $h_{\mathbf{c}}^{p, q}$. The Poincaré duality provides an isomorphism $H_{p}(\mathcal{M}) \sim H_{\mathbf{c}}^{4-p}(\mathcal{M})$, where $H_{p}(\mathcal{M})$ are the homology groups. Call $b_{p}$ their dimensions (the Betti numbers); then $b_{p}=b_{\mathbf{c}}^{4-p}$.

The fundamental topological invariants characterizing the gravitational instantons were recognized long time ago ([10], for a review see [19]) to be the Euler characteristic $\chi$ and the Hirzebruch signature $\tau$ of the base manifold.

The Euler characteristic is the alternating sum of the Betti numbers:

$$
\chi=\sum_{p=0}^{4}(-1)^{p} b_{p}=\sum_{p=0}^{4}(-1)^{p} b_{\mathbf{c}}^{4-p}=\sum_{p=0}^{4}(-1)^{p} b_{\mathbf{c}}^{p} .
$$

The Hirzebruch signature is the difference between the number of positive and negative eigenvalues of the quadratic form on $H_{\mathbf{c}}^{2}(\mathcal{M})$ given by the cup product $\int_{\mathcal{M}} \alpha \wedge \beta$, with $\alpha, \beta \in H_{\mathbf{c}}^{2}(\mathcal{M})$. That is, if $b_{\mathbf{c}}^{2(+)}$ and $b_{\mathbf{c}}^{2(-)}$ are the number of selfdual and anti-selfdual 2 -forms with compact support, $\tau=b_{\mathbf{c}}^{2(+)}-b_{\mathbf{c}}^{2(-)}$. At this point, we need two observations.

1. The HyperKähler forms $\Omega^{3}, \Omega^{ \pm}$, being covariantly constant, cannot be $\mathbf{L}^{2}$ if the space is non-compact

2. In the compact case they are the unique antiselfdual 2 -forms, so that $b^{2(-)}=3$, $b^{2(+)}=\tau+3$. Indeed from the expression of the Hirzebruch signature in terms of the Hodge numbers, $\tau=\sum_{p+q=0 \bmod 2}(-1)^{p} h^{p, q}$ (see [20, chap. 0, sec. 7]), using the consequences of the Calabi-Yau condition $c_{1}(\mathcal{M})=0 \Rightarrow h^{2,0}=h^{0,2}=1$ and the fact that $h^{0,0}=h^{2,2}=1$ we obtain $h^{1,1}=\tau+4$. Hence the cohomology in degree two splits as follows:

$$
\begin{array}{ccc}
h^{2,0} & h^{1,1} & h^{0,2} \\
1 & 1+(\tau+3) & 1
\end{array}
$$

This leads to the conclusion that $\Omega^{3} \in H^{1,1}$ and $\Omega^{ \pm} \in H^{2,0}$ (resp. $H^{0,2}$ ) are the unique antiselfdual two-forms.

In the non compact case, by the observation (1) the HyperKähler two-forms are deleted from the compact support cohomology groups. However the Hirzebruch signature is what it is, hence also other three selfdual two-forms have to be deleted as being non square-integrable, in order to maintain the value of $\tau$. 
The "Hodge diamonds" for the usual and $\mathbf{L}^{2}$ Dolbeaut cohomology groups are respectively given by:

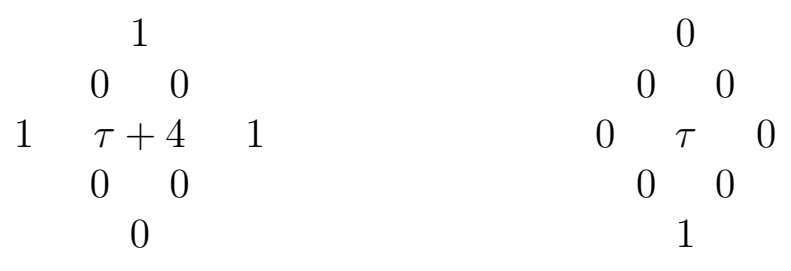

Note that, from eq.(8), $\chi=\tau+1$.

In the $(4,4)$ SCFT corresponding to a non-compact gravitational instanton we expect therefore to be able to distinguish four of the $\Psi_{A}$ as giving rise to "non-normalizable" deformations. We will see how this is realized in the case of ALE spaces.

$\underline{A L E \text { spaces }}$

The most natural gravitational analogues of the Yang-Mills instantons would be represented by Riemannian manifolds geodesically complete and such that

1. the curvature 2-form is (anti)selfdual;

2. the metric approaches the Euclidean metric at infinity; that is, in polar coordinates $(r, \boldsymbol{\Theta})$ on $\mathbf{R}^{4}$

$$
g_{\mu \nu}(r, \Theta)=\delta_{\mu \nu}+O\left(r^{-4}\right)
$$

This would agree with the "intuitive" picture of instantons as being localized in finite regions of space-time. The above picture is verified however only modulo an additional subtlety: the base manifold has a boundary at infinity $S^{3} / \Gamma, \Gamma$ being a finite group of identifications. "Outside the core of the instanton" the manifold looks like $\mathbf{R}^{4} / \Gamma$ instead of $\mathbf{R}^{4}$. This is the reason of the name given to these spaces: the asymptotic behaviour is only locally euclidean. The unique globally euclidean gravitational instanton is euclidean four-space itself.

This kind of behaviour is easily seen in the simplest of these metrics, the EguchiHanson metric 12]:

$$
d s^{2}=\frac{d r^{2}}{1-\left(\frac{a}{r}\right)^{4}}+r^{2}\left(\sigma_{x}^{2}+\sigma_{y}^{2}\right)+r^{2}\left[1-\left(\frac{a}{r}\right)^{4}\right] \sigma_{z}^{2},
$$

where $a$ is a real constant, $\sigma_{x}, \sigma_{y}, \sigma_{z}$ are Maurer-Cartan forms of $S U(2)$ realized in terms of the Euler angles given by the angles of the polar coordinates on $\mathbf{R}^{4} \theta, \phi, \psi$. By changing the radial coordinate: $u^{2}=r^{2}\left[1-\left(\frac{a}{r}\right)^{4}\right]$ the apparent singularity at $r=a$ is moved to $u=0$ :

$$
d s^{2}=\frac{d u^{2}}{\left[1+\left(\frac{a}{r}\right)^{4}\right]^{2}}+u^{2} \sigma_{z}^{2}+r^{2}\left(\sigma_{x}^{2}+\sigma_{y}^{2}\right)
$$


Since near $u=0 d s^{2} \simeq \frac{1}{4} d u^{2}+\frac{1}{4} u^{2}(d \psi+\cos \theta d \phi)^{2}+\frac{a^{2}}{4}\left(d \theta^{2}+\sin ^{2} \theta d \phi^{2}\right)$, at fixed $\theta, \phi$ the singularity in $d s^{2} \simeq \frac{1}{4}\left(d u^{2}+u^{2} d \psi^{2}\right)$ looks like the removable singularity due to the use of polar coordinates in $\mathbf{R}^{2}$, provided that $0 \leq \psi<2 \pi$, which is not the range assumed by the polar angle $\psi$ on $\mathbf{R}^{4}$; in this case the range is instead $0 \leq \psi<4 \pi$. Thus opposite points on the constant-radius slices are to be identified, and the boundary at infinity is $S^{3} / \mathbf{Z}_{2}$

Subsequent work, leading to the construction of the "multi-Eguchi-Hanson" metrics [21] and their reinterpretation in terms of a twistor construction [14], culminating with the papers by Kronheimer [15, 16], established the following picture.

Every ALE space is determined by its group of identifications $\Gamma$, which must be a finite Kleinian subgroup of $S U(2)$. Kronheimer described indeed manifolds having such a boundary; he showed that in principle an unique selfdual metric can be obtained for for each of these manifolds [15] and, moreover, that every selfdual metric approaching asymptotically the euclidean one can be recovered in such a manner [16]. The classification of $S U(2)$ finite subgroups is well-known, and is reviewed in the following section.

\section{The Kleinian subgroups of $S U(2)$}

The classification of the finite (Kleinian) subgroups of $S U(2)$ is a classical result of nineteenth century mathematics [22] and can be related in a one-to-one fashion to the twentieth century classification of simply laced Lie algebras (ADE classification), as much as to the two thousand years old Platonic classification of regular polygons, dihedra and polyhedra [23]. The explicit construction of the ALE manifolds as HyperKähler quotients obtained in 1989 by Kronheimer [15, 16] relies heavily on the algebraic structure of the Kleinian groups and puts into evidence the crucial identification between the most relevant topological number of the manifold, namely its Hirzebruch signature $\tau$ and the number $r$ of conjugacy classes of the finite group $\Gamma$. Furthermore it is this identification that provides a clue for the construction of the corresponding $(4,4)$ conformal field theories. Choosing complex coordinates $z_{1}=x-i y, z_{2}=t+i z$ on $\mathbf{R}^{4} \sim \mathbf{C}^{2}$, and representing a point $\left(z_{1}, z_{2}\right)$ by a quaternion (see sec. 4), the group $S O(4) \sim S U(2)_{L} \times S U(2)_{R}$, which is the isometry group of the sphere at infinity, acts on the quaternion by matrix multiplication:

$$
\left(\begin{array}{cc}
z_{1} & i \bar{z}_{2} \\
i z_{2} & \bar{z}_{1}
\end{array}\right) \longrightarrow M_{1} \cdot\left(\begin{array}{cc}
z_{1} & i \bar{z}_{2} \\
i z_{2} & \bar{z}_{1}
\end{array}\right) \cdot M_{2}
$$

the element $M \in S O(4)$ being represented as $\left(M_{1} \in S U(2)_{L}, M_{2} \in S U(2)_{R}\right)$. The group $\Gamma$ can be seen as a finite subgroup of $S U(2)_{L}$, acting on $\mathbf{C}^{2}$ in the natural way by its two-dimensional representation:

$$
\forall U \in \Gamma \subset S U(2), \quad U: \mathbf{v}=\left(\begin{array}{c}
z_{1} \\
z_{2}
\end{array}\right) \longrightarrow U \mathbf{v}=\left(\begin{array}{cc}
\alpha & i \beta \\
i \bar{\beta} & \bar{\alpha}
\end{array}\right)\left(\begin{array}{c}
z_{1} \\
z_{2}
\end{array}\right)
$$

In characterizing such finite subgroups it appears a Diophantine equation which is just the same one which is encountered in the classification of the possible simply laced 
Dynkin diagrams [24]. As a result, the possible finite subgroups of $S U(2)$ are organized in two infinite series and three exceptional cases; each subgroup $\Gamma$ is in correspondence with a simply laced Lie algebra $\mathcal{G}$, and we write $\Gamma(\mathcal{G})$ for it. One series is given by the cyclic subgroups groups of order $k+1$, related to $A_{k}$; the other series is that of the dihedral subgroups containing a cyclic subgroup of order $k$, related to $D_{k+2}$; the remaining three subgroups $\Gamma\left(E_{6}\right) \approx \mathcal{T}, \Gamma\left(E_{7}\right) \approx \mathcal{O}$ and $\Gamma\left(E_{8}\right) \approx \mathcal{I}$ have order 12,24 and 60, respectively, and they correspond to the binary extensions of the Tetrahedron, Octahedron and Icosahedron symmetry groups. More specifically, the ADE classification of $S U(2)$ finite subgroups is obtained by considering the "poles" $P_{i}$ identified by the subgroup $\Gamma$, where $P_{i}=\left\{\lambda v_{i}\right\} i=1,2$ is, by definition, the one-dimensional subspace of $\mathbf{C}^{2}$ identified by an eigenvector $v_{i}$ of some element $\gamma \in \Gamma$. Since each nontrivial element of the group $S U(2)$ has two distinct orthogonal eigenvectors, a finite subgroup $\Gamma$ of order $n$ singles out $2 n-2$ poles that are not necessarily distinct. (For instance, in the case of the $\mathbf{Z}_{n}$ subgroup generated by the rotation of an angle $\frac{2 \pi}{n}$ around some axis, all elements of the group single out the same pair of distinguished poles). If we call equivalent two poles $P_{i}$ and $P_{j}$ that are mapped one into the other by some elements $\gamma \in \Gamma\left(P_{i}=\gamma P_{j}\right)$, then we can distribute the $2 n-2$ poles into $r$ equivalence classes $\mathcal{C}_{\alpha}(\alpha=1, \ldots r)$. We name $m_{\alpha}$ the number of distinguished poles that belong to the class $\mathcal{C}_{\alpha}$ and $q_{\alpha}$ the total number of poles in $\mathcal{C}_{\alpha}$, counting as different also coincident poles. A simple argument for which we refer to [24] shows that $q_{\alpha}=m_{\alpha}\left(k_{\alpha}-1\right)$, where $k_{\alpha}$ is the order of the stability subgroup $K_{P}$ of any pole $P$ in $\mathcal{C}_{\alpha}$. Indeed, one sees that all equivalent poles have conjugate stability subgroups, so that their order depends only on the equivalence class. Furthermore, the stability subgroups $K_{P}$ are cyclic groups $K_{P} \approx \mathbf{Z}_{k_{\alpha}}$. Using this information, one immediately obtains

$$
2 n-2=\sum_{\alpha=1}^{r} m_{\alpha}\left(k_{\alpha}-1\right)
$$

Another simple argument shows that for each equivalence class $\mathcal{C}_{\alpha}$ we have $m_{\alpha} k_{\alpha}=n$. Indeed, it is sufficient to decompose the group $\Gamma=K_{P}+g_{1} K_{P}+\ldots+G_{s} K_{P}$ into cosets with respect to the stability subgroup $K_{P}$ of any pole $P$ in $\mathcal{C}_{\alpha}$, and to remark that the number $s$ of cosets is just equal to the number $m_{\alpha}$ of distinct poles equivalent to $P$. (By definition, $g_{i} P$ is different from $P$ and equivalent to $P$ ). Dividing eq.(15) by $n$ and using this information, we obtain the Diophantine equation leading to the ADE classification, namely

$$
2\left(1-\frac{1}{n}\right)=\sum_{\alpha=1}^{q}\left(1-\frac{1}{k_{\alpha}}\right) .
$$

Since by definition $k_{\alpha} \geq 2$, a little bit of elaboration of eq. (16) shows that it implies, as only possible cases $q=2$ or $q=3$. In the case $q=2$ eq. (16) reduces to

$$
\frac{1}{k_{1}}+\frac{1}{k_{2}}=\frac{2}{n}
$$




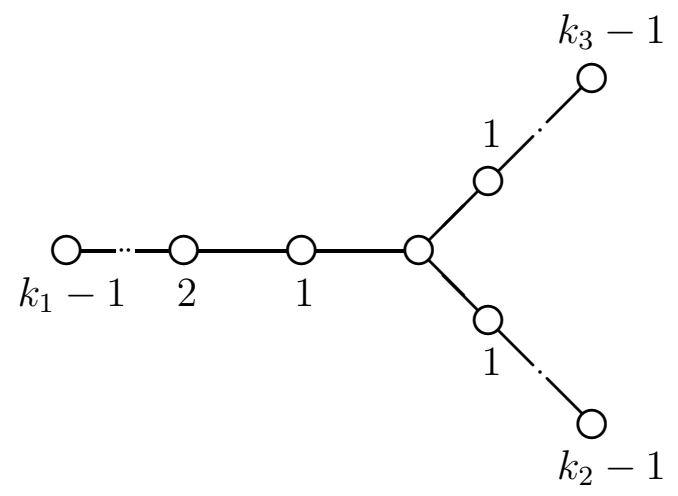

Figure 1: Interpretation of the numbers $k_{i}$

whose only solution is $k_{1} J=k_{2}=k+1$. This is the $A_{k}$ solution and the corresponding subgroup $\Gamma\left(A_{k}\right) \approx \mathbf{Z}_{k+1}$ is cyclic of order $k+1$, its elements being the matrices given in eq. (24) Indeed the two equivalence classes of poles are spanned by the two eigenvectors of the single $\mathbf{Z}_{k+1}$ generator that are common to all the group elements.

In the $q=3$ case the Diophantine equation (16) becomes:

$$
\frac{1}{k_{1}}+\frac{1}{k_{2}}+\frac{1}{k_{3}}=1+\frac{2}{n}
$$

admitting the infinite $D_{k+2}$ solution $k_{1}=k, k_{2}=k_{3}=2$ and the three exceptional $E_{6}, E_{7}$ and $E_{8}$ solutions respectively given by $k_{1}=3, k_{2}=3, k_{3}=2$ or $k_{1}=4, k_{2}=3, k_{3}=2$, or $k_{1}=5, k_{2}=3, k_{3}=2$. The reason why these solutions are associated with the names of the simply laced Lie algebras is that the numbers $k_{i}-1$ can be interpreted as the number of dots in each simple chain departing from the dot at the center of a node in a corresponding Dynkin diagram (see 3).

The group $\Gamma\left(D_{k+2}\right)$ is the dihedral subgroup. Its order is

$$
\left|\Gamma\left(D_{k+2}\right)\right|=4 k
$$

and it contains a cyclic subgroup of order $k$ that we name $K$. Its index in $\Gamma\left(D_{k+2}\right)$ is two. The elements of $\Gamma\left(D_{k+2}\right)$ that are not in $K$ are of period equal to two since $k_{2}=k_{3}=2$. Altogether the elements of the dihedral group are the matrices given in eq.(33). The remaining three subgroups $\Gamma\left(E_{6}\right) \approx \mathcal{T}, \Gamma\left(E_{7}\right) \approx \mathcal{O}$ and $\Gamma\left(E_{8}\right) \approx \mathcal{I}$ can be similarly described.

Let us now consider more closely the algebraic structure of these groups, in particular in relation with their conjugacy classes, whose number equals the Hirzebruch signature of the corresponding ALE manifold. We begin by fixing notations. For any finite group $\Gamma$ we denote by $K_{i},(i=1, \ldots, r)$ the conjugacy classes of its elements: iff $\gamma_{1} \gamma_{2} \in$ $K_{i}$ then $\exists h \in \Gamma / \gamma_{1}=h^{-1} \gamma_{2} h$ and we name $g_{i}=\left|K_{i}\right|$ the order of the $i$-th conjugacy class. One obviously has $|\Gamma| J=g=\sum_{i=1}^{r} g_{i}$. For any representation $D$ of $\Gamma$, we denote by $\left\{\chi_{1}^{(D)}, \ldots \ldots, \chi_{r}^{(D)}\right\}$ its character vector where $\chi_{i}^{(D)}=\operatorname{Tr}\left(D\left(\gamma_{i}\right) J\right)$ is the trace of any representative of the $i$-th class. As it is well-known the number of conjugacy classes $r$ equals the number of irreducible representations: we name these latter $D_{\mu}$ 
$(\mu=1, \ldots, r)$. The square matrix $\chi_{i}^{\mu}$ whose rows are the character vectors of the irreducible representations is named the character table. It satisfies the orthogonality relations

$$
\sum_{\mu=1}^{r} \chi_{i}^{\mu} \chi_{j}^{\mu}=\frac{g}{g_{i}} \delta_{i j}, \quad \sum_{i=1}^{r} \chi_{i}^{\mu} \chi_{i}^{\nu}=g \delta^{\mu \nu}
$$

that imply the following sum rule for the dimensions $n_{\mu}=\operatorname{Tr} D_{\mu}(\mathbf{1})$ of the irreducible representations:

$$
\sum_{\mu=1}^{r} n_{\mu}^{2}=g=|\Gamma| .
$$

Relevant to our use of the Kleinian groups are also the $g$-dimensional regular representation $R$, whose basis vectors $e_{\gamma}$ are in one-to-one correspondence with the group elements $\gamma$ and transform as

$$
R(\gamma) e_{\delta}=e_{\gamma \cdot \delta} \quad \forall \gamma, \delta \in \Gamma
$$

and the 2-dimensional defining representation $\mathcal{Q}$ which is obtained by regarding the group $\Gamma$ as an $S U(2)$ subgroup [that is, $\mathcal{Q}$ is the representation which acts in eq.(14)]. The character table allows to reconstruct the decomposition coefficients of any representation along the irreducible representations. If $D=\bigoplus_{\mu=1}^{r} a_{\mu} D_{\mu}$ we have $a_{\mu}=$ $\frac{1}{g} \sum_{i=1}^{r} g_{i} \chi_{i}^{(D)} \chi_{i}^{(\mu) \star}$. For the Kleinian groups $\Gamma$ a particularly important case is the decomposition of the tensor product of an irreducible representation $D_{\mu}$ with the defining 2-dimensional representation $\mathcal{Q}$. It is indeed at the level of this decomposition that the relation between these groups and the simply laced Dynkin diagrams is more explicit [25]. Furthermore this decomposition plays a crucial role in the explicit construction of the ALE manifolds [15]. Setting

$$
\mathcal{Q} \otimes D_{\mu}=\bigoplus_{\nu=0}^{r} A_{\mu \nu} D_{\nu}
$$

where $D_{0}$ denotes the identity representation, one finds that the matrix $\bar{c}_{\mu \nu}=2 \delta_{\mu \nu}-A_{\mu \nu}$ is the extended Cartan matrix relative to extended Dynkin diagram corresponding to the given group. We remind the reader the the extended Dynkin diagram of any simply laced Lie algebra is obtained by adding to the dots representing the simple roots $\left\{\alpha_{1} \ldots \ldots \alpha_{r}\right\}$ an additional dot (marked black in Fig.s 3, 3) representing the negative of the highest root $\alpha_{0}=\sum_{i=1}^{r} n_{i} \alpha_{i}$ ( $n_{i}$ are the Coxeter numbers). We see thus an correspondence between the non-trivial conjugacy classes $K_{i}$ or equivalently the non-trivial irrepses of the group $\Gamma(\mathcal{G})$ and the simple roots of $\mathcal{G}$. In this correspondence, as we have already remarked the extended Cartan matrix provides us with the Clebsch-Gordan coefficients (23), while the Coxeter numbers $n_{i}$ express the dimensions of the irreducible representations. All these informations are summarized in Fig.s 2,3 where the numbers $n_{i}$ are attachedto each of the dots: the number 1 is attached to the extra dot because it stands for the identity representation.

Let us now briefly consider the structure of the irreducible representations and of the character tables. 


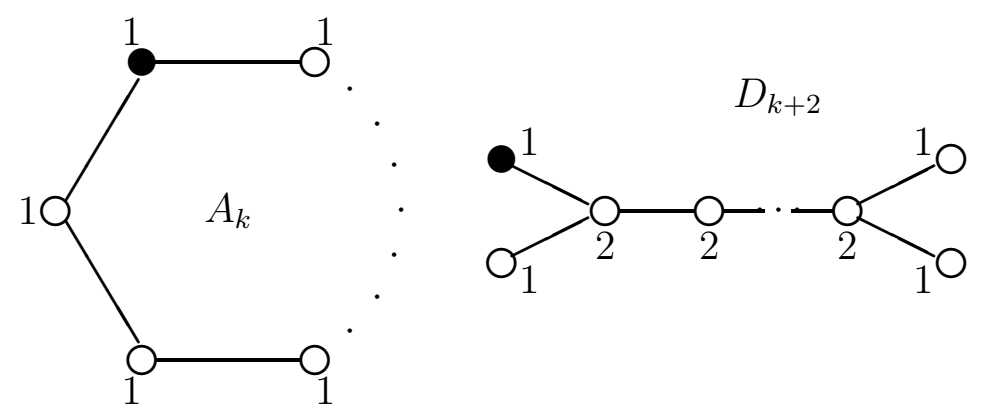

Figure 2: Extended Dynkin diagrams of the infinite series

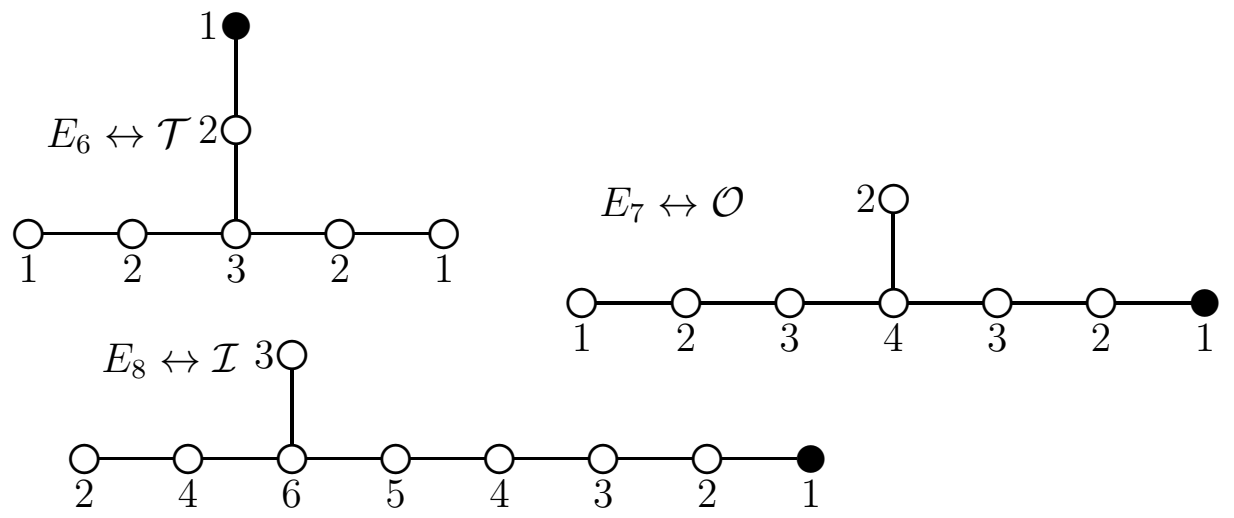

Figure 3: Exceptional extended Dynkin diagrams

$\underline{A_{k} \text {-series }}$ In this case the defining 2-dimensional representation $\mathcal{Q}$ is given by the matrices

$$
\gamma_{l} \in \Gamma\left(A_{k}\right) \quad ; \quad \gamma_{l}=\mathcal{Q}_{l} \stackrel{\text { def }}{=}\left(\begin{array}{cc}
e^{2 \pi i l /(k+1)} & 0 \\
0 & e^{-2 \pi i l /(k+1}
\end{array}\right)\{l=1, \ldots ., k\} .
$$

It is not irreducible since all irreducible representations are one-dimensional as one sees from Fig. 3 . In the $j$-th irreducible representation the $1 \times 1$-matrix representing the $l$-th element of the group is

$$
D^{(j)}\left(e_{l}\right)=\nu^{j l} \quad ; \quad \text { where } \nu=\exp \frac{2 \pi i}{k} .
$$

The $(k+1) \times(k+1)$ array of phases $\nu^{j l}$ appearing in the above equation is the character table. Given the $\mathbf{C}^{2}$ carrier space of the defining representation [see eq.s (14)] it is fairly easy to construct three algebraic invariants, namely

$$
\begin{aligned}
z & =z_{1} z_{2} \\
x & =\left(z_{1}\right)^{k+1} \\
y & =\left(z_{2}\right)^{k+1}
\end{aligned}
$$


that satisfy the polynomial relation

$$
W_{A_{k}}(x, y, z) \stackrel{\text { def }}{=} x y-z^{k+1}=0
$$

As stressed in the introduction the polynomial constraint $W_{\Gamma}(x, y, z)=0$ plays an important role in the construction of the ALE manifolds and of the associated $(4,4)$ conformal field-theory. Indeed, as we are going to see in the next sections, the vanishing locus in $\mathbf{C}^{3}$ of the potential $W_{\Gamma}(x, y, z)$ coincides with the space of equivalence classes $\mathrm{C}^{2} / \Gamma$, that is with the singular orbifold limit of the self-dual manifold $\mathcal{M}_{\Gamma}$. According to the standard procedure of deforming singularities [26] there is a corresponding family of smooth manifolds $\mathcal{M}_{\Gamma}\left(t_{1}, t_{2}, \ldots . ., t_{r}\right)$ obtained as the vanishing locus $Z_{0} \in \mathbf{C}^{3}$ of a deformed potential:

$$
\tilde{W}_{\Gamma}\left(x, y, z ; t_{1}, t_{2}, \ldots . ., t_{r}\right)=W_{\Gamma}(x, y, z)+\sum_{i=1}^{r} t_{i} \mathcal{P}^{(i)}(x, y, z)
$$

where $t_{i}$ are complex numbers (the moduli of the complex structure of $\left.\mathcal{M}_{\Gamma}\right)$ and $\mathcal{P}^{(i)}(x, y, z)$ is a basis spanning the chiral ring

$$
\mathcal{R}=\frac{\mathbf{C}[x, y, z]}{\partial W}
$$

of polynomials in $x, y, z$ that do not vanish upon use of the vanishing relations $\partial_{x} W=$ $\partial_{y} W=\partial_{z} W=0$. It is a matter of fact that the dimension of this chiral ring $|\mathcal{R}|$ is precisely equal to the number of non-trivial conjugacy classes (or of non trivial irreducible representations) of the finite group $\Gamma$. From the geometrical point of view this implies an identification between the number of complex structure deformations of the ALE manifold and the number $r$ of non-trivial conjugacy classes discussed above. From the $(4,4) \mathrm{CFT}$ viewpoint this relation implies that $r$ must also be the number of short representations, whose last components (moduli operators) can be used to deform the theory. In other words we have $\tau=r$, where $\tau$ is the Hirzebruch signature. Indeed in the language of algebraic geometry the singular orbifold $\mathbf{C}^{2} / \Gamma$ corresponding to the vanishing locus $Z_{0}$ of the potential $W$ admits an equivariant minimal resolutions of singularity $Z \stackrel{\lambda}{\longrightarrow} Z_{0}$, where $Z$ is a smooth variety, $\lambda$ is an isomorphism outside the singular point $\{0\} \in Z_{0}$ and it is a proper map such that $\lambda^{-1}\left(Z_{0}-0\right)$ is dense in $Z$. The fundamental fact is that the exceptional divisor $\lambda^{-1}(0) \subset Z$ consists of a set of irreducible curves $c_{\alpha}, \alpha=1, \ldots r$ which can be put in correspondence with the vertices of the Dynkin diagram (the non-extended one) of the simple Lie Algebra corresponding to $\Gamma$ as above. Each $c_{\alpha}$ is isomorphic to a copy of $\mathbf{C P}^{1}$; the intersection matrix of these non-trivial two-cycles is the negative of the Cartan matrix:

$$
c_{\alpha} \cdot c_{\beta}=\bar{c}_{\alpha \beta} .
$$

Kronheimer construction, described in section $₫$, shows that the base manifold $\mathcal{M}$ of an ALE space is diffeomorphic to the space $Z$ supporting the resolution of the orbifold 
$Z_{0} \sim \mathbf{C}^{2} / \Gamma$, see section 4 . Therefore the equation (30) applies to the generators of the second homology group of $\mathcal{M}$. In particular we see that

$$
\begin{aligned}
\tau & =\operatorname{dim}_{\mathbf{c}}^{2}(X)=\operatorname{dimH}_{2}(X)= \\
& =\text { rank of the corresponding Lie Algebra }= \\
& =\# \text { non trivial conj. classes in } \Gamma=|\mathcal{R}|
\end{aligned}
$$

The chiral ring of the potential (39) has $k+2$ elements just matching the number of non-trivial conjugacy classes. According to our previous discussion $k+2$ will also be the number of short representations in the corresponding $(4,4)$ conformal field-theory.

These results are summarized in Table 2 which compares the algebraic information on the Kleinian group structure with the classical results on the topology of the ALE manifolds obtained in the late seventies by means of the index theorems (see [19]). Indeed in the last columns of Table 22 we list the Hirzebruch signature $\tau$, the Euler character $\chi$ and the spin $3 / 2$ index $I_{3 / 2}$. As one sees, the Hirzebruch signature is always equal to the dimension of the chiral ring, which also equals the number of conjugacy classes of the Kleinian group. The spin $3 / 2$ index counts the normalizable gravitino zero-modes and turns out to be equal to $2 \tau=|\mathcal{R}|$. This is in agreement with the results of [⿴囗十 .

$D_{k+2}$-series Abstractly the binary extension $D_{k+2}$ of the dihedral group could be described introducing the generators $\mathcal{A}, \mathcal{B}, \mathcal{Z}$ and setting the relations:

$$
\begin{aligned}
& \mathcal{A}^{k}=\mathcal{B}^{2}=(\mathcal{A B})^{2}=\mathcal{Z} \\
& \mathcal{Z}^{2}=1
\end{aligned}
$$

The $4 k$ elements of the group are given by the following matrices:

$$
\begin{aligned}
& F_{l}=\left(\begin{array}{cc}
e^{i l \pi / k} & 0 \\
0 & e^{-i l \pi / k}
\end{array}\right) \quad ; \quad(l=0,1,2, \ldots . ., 2 k-1) \\
& G_{l}=\left(\begin{array}{cc}
0 & i e^{-i l \pi / k} \\
i e^{i l \pi / k} & 0
\end{array}\right) \quad ; \quad(l=0,1,2, \ldots . ., 2 k-1)
\end{aligned}
$$

In terms of them the generators are identified as follows:

$$
F_{0}=1 ; F_{1}=\mathcal{A} ; F_{k}=\mathcal{Z} ; \quad G_{0}=\mathcal{B}
$$

There are exactly $r=k+3$ conjugacy classes

1. $K_{e}$ contains only the identity $F_{0}$

2. $K_{Z}$ contains the central extension $\mathcal{Z}$

3. $K_{\text {Geven }}$ contains the elements $G_{2 \nu}(\nu=1, \ldots, k-1)$

4. $K_{G \text { odd }}$ contains the elements $G_{2 \nu+1}(\nu=1, \ldots, k-1)$ 
5. the $k-1$ classes $K_{F_{\mu}}$ : each of these classes contains the pair of elements $F_{\mu}$ and $F_{2 k-\mu}$ for $(\mu=1, \ldots, k-1)$.

Correspondingly the $D_{k+2}$ group admits $k+3$ irreducible representations 4 of which are 1-dimensional while $k-1$ are 2-dimensional. We name them as follows:

$$
\left\{\begin{array}{l}
D_{e} ; D_{Z} ; D_{\text {Geven }} ; D_{G \text { odd }} ; 1 \text {-dimensional } \\
D_{F_{1}} ; \ldots \ldots . . ; D_{F_{k-1}} ; 2 \text {-dimensional }
\end{array}\right.
$$

The combinations of the $\mathbf{C}^{2}$ vector components $\left(z_{1}, z_{2}\right)$ that transform in the four 1dimensional representations are easily listed:

$$
\begin{aligned}
& D_{e} \quad \longrightarrow \quad\left|z_{1}\right|^{2}+\left|z_{2}\right|^{2} \\
& D_{Z} \quad \longrightarrow \quad z_{1} z_{2} \\
& D_{\text {Geven }} \longrightarrow z_{1}^{k}+z_{2}^{k} \\
& D_{\text {Godd }} \quad \longrightarrow \quad z_{1}^{k}-z_{2}^{k} \text {. }
\end{aligned}
$$

The matrices of the $k-1$ two-dimensional representations are obtained in the following way. In the $D F_{s}$ representation, $s=1, \ldots k-1$, the generator $\mathcal{A}$, namely the group element $F_{1}$, is represented by the matrix $F_{s}$. The generator $\mathcal{B}$ is instead represented by $(i)^{s-1} G_{0}$ and the generator $\mathcal{Z}$ is given by $F_{s k}$, so that:

$$
\begin{aligned}
D F_{s}\left(F_{j}\right) & =F_{s j} \\
D F_{s}\left(G_{j}\right) & =(i)^{s-1} G_{s j} .
\end{aligned}
$$

The character table is immediately obtained and it is displayed in Table 1. Using the one-dimensional representations (36) we can define the following invariants:

$$
\begin{aligned}
z & =-\left(z_{1} z_{2}\right)^{2} \\
x & =\frac{i}{2} z_{1} z_{2}\left(z_{1}^{2 k}-(-1)^{k} z_{2}^{2 k}\right) \\
y & =\frac{i}{2}\left(z_{1}^{2 k}+(-1)^{k} z_{2}^{2 k}\right)
\end{aligned}
$$

that, in the $D_{k+2}$ case, fulfill the relation

$$
W_{D_{k+2}}(x, y, z) \stackrel{\text { def }}{=} x^{2}+y^{2} z+z^{k+1}=0
$$

the analogue of the relation (27) obtained in the $A_{k}$ case. The chiral ring of the potential (39) has $k+2$ elements just matching the number of non-trivial conjugacy classes. According to our previous discussion $k+2$ will also be the number of short representations in the corresponding $(4,4)$ conformal field-theory.

In a similar way one can retrieve the structure of the irreducible representations and the potential also for the three exceptional groups $\mathcal{T}, \mathcal{O}$ and $\mathcal{I}$. 
Table 1: Character table of the Group $D_{k+2}$

\begin{tabular}{||c||c|c|c|c|c|c|c||}
\hline$\cdot$ & $K E$ & $K Z$ & $K G_{e}$ & $K G_{o}$ & $K F_{1}$ & $\cdots$ & $K F_{k-1}$ \\
\hline \hline$D E$ & 1 & 1 & 1 & 1 & 1 & $\cdots$ & 1 \\
\hline$D Z$ & 1 & 1 & -1 & -1 & 1 & $\cdots$ & 1 \\
\hline$D G_{e}$ & 1 & $(-1)^{k}$ & $i^{k}$ & $-i^{k}$ & $(-1)^{1}$ & $\cdots$ & $(-1)^{k-1}$ \\
\hline$D G_{o}$ & 1 & $(-1)^{k}$ & $-i^{k}$ & $i^{k}$ & $(-1)^{1}$ & $\cdots$ & $(-1)^{k-1}$ \\
\hline$D F_{1}$ & 2 & $(-2)^{1}$ & 0 & 0 & $2 \operatorname{Cos} \frac{\pi}{k}$ & $\cdots$ & $2 \operatorname{Cos} \frac{(k-1) \pi}{k}$ \\
\hline$\vdots$ & $\vdots$ & $\vdots$ & $\vdots$ & $\vdots$ & $\vdots$ & $\ddots$ & $\vdots$ \\
\hline$D F_{k-1}$ & 2 & $(-2)^{k-1}$ & 0 & 0 & $2 \operatorname{Cos} \frac{(k-1) \pi}{k}$ & $\cdots$ & $2 \operatorname{Cos} \frac{(k-1)^{2} \pi}{k}$ \\
\hline
\end{tabular}

Table 2: KLEINIAN GROUP versus ALE MANIFOLD properties

\begin{tabular}{||l|c|c|c|c|c|c||}
\hline$\Gamma$. & $W(x, y, z)$ & $\mathcal{R}=\frac{\mathbf{C}[x, y, z]}{\partial W}$ & $|\mathcal{R}|$ & $\# c . c$. & $\tau \equiv \chi-1$ & $I_{3 / 2}$ \\
\hline \hline$A_{k}$ & $x y-z^{k+1}$ & $\begin{array}{c}\{1, z, . . \\
\left.., z^{k-1}\right\}\end{array}$ & $k$ & $k+1$ & $k$ & $2 k$ \\
\hline$D_{k+2}$ & $x^{2}+y^{2} z+z^{k+1}$ & $\begin{array}{c}\left\{1, y, z, y^{2},\right. \\
\left.z^{2}, \ldots, z^{k-1}\right\}\end{array}$ & $k+2$ & $k+3$ & $k+2$ & $\begin{array}{r}2 k \\
+4\end{array}$ \\
\hline$E_{6}=$ & $x^{2}+y^{3}+z^{4}$ & $\begin{array}{c}\{1, y, z, \\
\left.y z, z^{2}, y z^{2}\right\}\end{array}$ & 6 & 7 & 6 & 12 \\
$\mathcal{T}$ & & $\begin{array}{c}\left\{1, y, z, y^{2},\right. \\
\left.z^{2}, y z, y^{2} z\right\}\end{array}$ & 7 & 8 & 7 & 14 \\
\hline$E_{7}=$ & $x^{2}+y^{3}+y z^{3}$ & & & & \\
\hline $\mathcal{O}$ & & $\begin{array}{c}\left\{1, y, z, z^{2}, y z,\right. \\
\left.z^{3}, y z^{2}, y z^{3}\right\}\end{array}$ & 8 & 9 & 8 & 16 \\
$\mathcal{I}$ & $x^{2}+y^{3}+z^{5}$ & & & \\
\hline
\end{tabular}




\section{Number of parameters in the ALE metrics}

The number of parameters in a general metric $g_{\mu \nu}$, a part from the breathing mode, equals the number of zero modes of the Lichnerowitz operator. These modes modes are represented by symmetric, traceless, harmonic tensors $\delta g_{\mu \nu}$ that in four dimensions can be obtained as

$$
\delta g_{\mu \nu}=s_{\mu}^{\rho} a_{\rho \nu},
$$

$s_{\mu \nu}, a_{\mu \nu}$ being the components of a selfdual (resp. antiselfdual) harmonic two-form. For HyperKähler four-manifolds, the number of such modes is clearly

$$
\text { \# traceless defs. }=b^{2(+)} b^{2(-)}=3 b^{2(-)} .
$$

A deformation $\delta g_{\mu \nu}$ is called normalizable ( $\mathbf{L}^{2}$-integrable) if

$$
\int_{\mathcal{M}} g^{\mu \nu} g^{\rho \sigma} \delta g_{\mu \rho} \delta g_{\nu \sigma} \leq \infty .
$$

The above deformations, eq.(40), are not normalizable when they decrease at radial infinity less strongly than $r^{-4}$. Adding such a $\delta g_{\mu \nu}$ to $g_{\mu \nu}$ would destroy the asymptotic behaviour (10). In (40) the anti-selfdual forms (the HyperKähler forms) are certainly non-normalizable; being covariantly constant, they tend to a constant at infinity. Thus the behaviour of $\delta g_{\mu \nu}=s_{\mu}^{\rho} a_{\rho \nu}$ at infinity is determined by the behaviour of $s_{\mu}^{\rho}$. The diamonds (9) show that the bad-behaved self-dual two-forms $s_{\mu}^{\rho}$ are three, so we get

$$
\text { \# traceless } \mathbf{L}^{2} \text { deformations }=3 \tau \text {. }
$$

We will see that this number has a particularly clear origin in the construction of the ALE spaces of section 4 . However one still has to disregard those deformations that can be readsorbed by means of diffeomorphisms. One must not consider zeromodes of the form $\delta g_{\mu \nu}=\nabla_{(\mu} \xi_{\nu)}$ for some vector field $\xi^{\mu}$. As shown by Hawking and Pope in [13], any such vector field $\xi^{\mu}$ should tend to one of the $S O(4)$ Killing vectors of the $S^{3}$-boundary that commute with the action of $\Gamma$, given in eq.(13,23). The generators of $S U(2)_{R}$ survive for all the possible groups $\Gamma$; in the case $A_{k-1}, k>2$ (Multi-Eguchi-Hanson metric) also the diagonal generator of $S U(2)_{L}$ commutes with $A_{k-1}$, and in the Eguchi-Hanson case $\left(A_{1}\right)$ all the six generators of $S O(4)$ commute with $C_{2}$.

Of course, we must exclude the true Killing vectors of the ALE metric (that, by definition, do not give rise to any deformation). The ALE metrics admit one Killing vector in all cases except the Eguchi-Hanson instanton, which have four of them, as can be seen also from the explicit form of the metric, eq.(11). Summarizing, we have:

\begin{tabular}{c|c|c|c|c|c|c} 
& $C_{2}(\mathrm{E}-\mathrm{H})$ & $A_{k-1}$ & $D_{k+2}$ & $E_{6}$ & $E_{7}$ & $E_{8}$ \\
\hline \# of defs. & 1 & $3 k-6$ & $3 k+4$ & 16 & 19 & 22
\end{tabular}




\section{Construction of ALE spaces}

HyperKähler quotients

Consider a compact Lie group $G$ acting on a HyperKähler manifold $\mathcal{S}$ of real dimension $4 n$ by means of Killing vector fields $\mathbf{X}$ holomorphic with respect to the three complex structures of $\mathcal{S}$; then these vector fields preserve also the HyperKähler forms Ð:

$$
\left.\begin{array}{l}
\mathcal{L}_{\mathbf{X}} g=0 \leftrightarrow \nabla_{(\mu} X_{\nu)}=0 \\
\mathcal{L}_{\mathbf{X}} \mathcal{J}^{i}=0, i=1,2,3
\end{array}\right\} \Rightarrow 0=\mathcal{L}_{\mathbf{X}} \Omega^{i}=i_{\mathbf{X}} d \Omega^{i}+d\left(i_{\mathbf{X}} \Omega^{i}\right)=d\left(i_{\mathbf{X}} \Omega^{i}\right)
$$

If $\mathcal{S}$ is simply connected, $d\left(i_{\mathbf{X}} \Omega^{i}\right)=0$ implies the existence of three functions $\mu_{i}^{\mathbf{X}}$ such that $d \mu_{i}^{\mathbf{X}}=i_{\mathbf{x}} \Omega^{i}$. The functions $\mu_{i}^{\mathbf{X}}$ are defined up to a constant, which can be arranged so to make them equivariant: $\mathbf{X} \mu_{i}^{\mathbf{Y}}=\mu_{i}^{[\mathbf{X}, \mathbf{Y}]}$.

The $\left\{\mu_{i}^{\mathbf{X}}\right\}$ constitute then a momentum map. This can be regarded as a map $\mu: \mathcal{S} \rightarrow$ $\mathbf{R}^{3} \otimes \mathcal{G}^{*}$, where $\mathcal{G}^{*}$ denotes the dual of the Lie algebra $\mathcal{G}$ of the group $G$. Indeed let $x \in \mathcal{G}$ be the element corresponding to the Killing vector $\mathbf{X}$; then for a given $m \in \mathcal{S}, \mu_{i}(m)$ : $x \longmapsto \mu_{i}^{\mathbf{X}}(m) \in \mathbf{C}$ is a linear functional on $\mathcal{G}$. In practice, expanding $\mathbf{X}=X_{A} \mathbf{k}^{A}$ in a basis of Killing vectors $\mathbf{k}^{A}$ such that $\left[\mathbf{k}^{A}, \mathbf{k}^{B}\right]=C^{A B}{ }_{C} \mathbf{k}^{C}$, where $C^{A B}{ }_{C} \mathbf{k}^{C}$ are the structure constants of $\mathcal{G}$, we have also $\mu_{i}^{\mathbf{X}}=X_{A} \mu_{i}^{A}, i=1,2,3$; the $\left\{\mu_{i}^{A}\right\}$ are the components of the momentum map.

The HyperKähler quotient 27] provides a way to construct from $\mathcal{S}$ a lower-dimensional HyperKähler manifold $\mathcal{M}$, as follows. Let $\mathcal{Z} \subset \mathcal{G}^{*}$ be the dual of the centre of $\mathcal{G}$. For each $\zeta \in \mathbf{R}^{3} \otimes \mathcal{Z}$ the level set of the momentum map

$$
\mathcal{N} \equiv \bigcap_{i} \mu_{i}^{-1}\left(\zeta^{i}\right) \subset \mathcal{S}
$$

which has dimension $\operatorname{dim} \mathcal{N}=\operatorname{dim} \mathcal{S}-3 \operatorname{dim} G$, is left invariant by the action of $G$, due to the equivariance of $\mu$. It is thus possible to take the quotient

$$
\mathcal{M}=\mathcal{N} / G
$$

$\mathcal{M}$ is a manifold of $\operatorname{dimension} \operatorname{dim} \mathcal{M}=\operatorname{dim} \mathcal{S}-4 \operatorname{dim} G$ as long as the action of $G$ on $\mathcal{N}$ has no fixed points. The three two-forms $\rho^{i}$ on $\mathcal{M}$, defined naturally via the restriction to $\mathcal{N} \subset \mathcal{S}$ of the $\Omega^{i}$ and the quotient projection from $\mathcal{N}$ to $\mathcal{M}$, turn out to be actually HyperKähler forms on $\mathcal{M}$.

For future use, it is important to note that, once chosen $\mathcal{J}^{3}$ as the preferred complex structure, the momentum maps $\mu_{ \pm}=\mu_{1} \pm i \mu_{2}$ are holomorphic (resp. antiholomorphic).

The standard use of the HyperKähler quotient is that of obtaining non trivial HyperKähler manifolds starting from a flat $4 n$ real-dimensional manifold $\mathbf{R}^{4 n}$ acted on by a suitable group $\mathrm{G}$ generating triholomorphic isometries [27, 28]. This is the way it is used also in the construction of ALE manifolds.

\footnotetext{
${ }^{1} \mathcal{L}_{\mathbf{x}}$ and $i_{\mathrm{x}}$ denote respectively the Lie derivative along the vector field $\mathbf{X}$ and the contraction (of forms) with it.
} 
The manifold $\mathbf{R}^{4 n}$ can be given a quaternionic structure, and the corresponding quaternionic notation is sometimes convenient. For $n=1$ one has the flat quaternionic space $\mathbf{H} \stackrel{\text { def }}{=}\left(\mathbf{R}^{4},\left\{J^{i}\right\}\right)$. We represent its elements

$$
q \in \mathbf{H}=x+i y+j z+k t=x^{0}+x^{i} J^{i}, \quad x, y, z, t \in \mathbf{R}
$$

realizing the quaternionic structures $J^{i}$ by means of Pauli matrices: $J^{i}=i\left(\sigma^{i}\right)^{T}$. Thus

$$
q=\left(\begin{array}{cc}
u & i \bar{v} \\
i v & \bar{u}
\end{array}\right) \quad \longrightarrow \quad \bar{q}=\left(\begin{array}{cc}
\bar{u} & -i \bar{v} \\
-i v & u
\end{array}\right)
$$

where $u=x^{0}+i x^{3}$ and $v=x^{1}+i x^{2}$. The euclidean metric on $\mathbf{R}^{4}$ is retrieved as $d \bar{q} \otimes d q=d s^{2} \mathbf{1}$. The HyperKähler forms are grouped into a quaternionic two-form

$$
\Theta=d \bar{q} \wedge d q \stackrel{\text { def }}{=} \Omega^{i} J^{i}=\left(\begin{array}{cc}
i \Omega^{3} & i \Omega^{+} \\
i \Omega^{-} & -i \Omega^{3}
\end{array}\right)
$$

For generic $n$, we have the space $\mathbf{H}^{n}$, of elements

$$
q=\left(\begin{array}{cc}
u^{a} & i \bar{v}^{a} \\
i v_{a} & \bar{u}_{a}
\end{array}\right) \quad \longrightarrow \quad \bar{q}=\left(\begin{array}{cc}
\bar{u}_{a} & -i \bar{v}^{a} \\
-i v_{a} & u^{a}
\end{array}\right) \quad \begin{aligned}
& u^{a}, v_{a} \in \mathbf{C}^{n} \\
& a=1, \ldots n
\end{aligned}
$$

Thus $d \bar{q} \otimes d q=d s^{2} \mathbf{1}$ gives $d s^{2}=d \bar{u}_{a} \otimes d u^{a}+d \bar{v}^{a} \otimes d v_{a}$ and the HyperKähler forms are grouped into the obvious generalization of the quaternionic two-form eq.(47): $\Theta=$ $\sum_{a=1}^{n} d \bar{q}_{a} \wedge d q^{a}=\Omega^{i} J^{i}$, leading to $\Omega^{3}=2 i \partial \bar{\partial} K$ where the Kähler potential $K$ is $K=$ $\frac{1}{2}\left(\bar{u}_{a} u^{a}+\bar{v}^{a} v_{a}\right)$, and to $\Omega^{+}=2 i d u^{a} \wedge d v_{a}, \Omega^{-}=\left(\Omega^{+}\right)^{*}$.

Let $\left(T_{A}\right)_{b}^{a}$ be the antihermitean generators of a compact Lie group $\mathrm{G}$ in its $n \times n$ representation. A triholomorphic action of $G$ on $\mathbf{H}^{n}$ is realized by the Killing vectors of components

$$
X_{A}=\left(\hat{T}_{A}\right)_{b}^{a} q^{b} \frac{\partial}{\partial q^{a}}+\bar{q}_{b}\left(\hat{T}_{A}\right)_{a}^{b} \frac{\partial}{\partial \bar{q}_{a}} \quad ; \quad\left(\hat{T}_{A}\right)_{b}^{a}=\left(\begin{array}{cc}
\left(T_{A}\right)_{b}^{a} & \mathbf{0} \\
\mathbf{0} & \left(T_{A}^{*}\right)_{b}^{a}
\end{array}\right) .
$$

Indeed one has $\mathcal{L}_{\mathbf{x}} \Theta=0$. The corresponding components of the momentum map are:

$$
\mu^{A}=\bar{q}^{a}\left(\begin{array}{cc}
\left(T_{A}\right)_{b}^{a} & \mathbf{0} \\
\mathbf{0} & \left(T_{A}^{*}\right)_{b}^{a}
\end{array}\right) q^{b}+\left(\begin{array}{cc}
c & \bar{b} \\
b & -i c
\end{array}\right)
$$

where $c \in \mathbf{R}, b \in \mathbf{C}$ are constants.

\section{$\underline{\text { Kronheimer construction }}$}

The HyperKähler quotient is performed on a suitable flat HyperKähler space $\mathcal{S}$ that now we define. Given any finite subgroup of $S U(2), \Gamma$, consider a space $\mathcal{P}$ whose elements are two-vectors of $|\Gamma| \times|\Gamma|$ complex matrices: $p \in \mathcal{P}=(A, B)$. The action of an element $\gamma \in \Gamma$ on the points of $\mathcal{P}$ is the following:

$$
\left(\begin{array}{l}
A \\
B
\end{array}\right) \quad \stackrel{\gamma}{\longrightarrow}\left(\begin{array}{cc}
u_{\gamma} & i \bar{v}_{\gamma} \\
i v_{\gamma} & \bar{u}_{\gamma}
\end{array}\right)\left(\begin{array}{l}
R(\gamma) A R\left(\gamma^{-1}\right) \\
R(\gamma) B R\left(\gamma^{-1}\right)
\end{array}\right)
$$


where the twodimensional matrix in the r.h.s. is the realization of $\gamma$ in the defining representation $\mathcal{Q}$ of $\Gamma$, while $R(\gamma)$ is the regular, $|\Gamma|$-dimensional representation, defined in section 3. This transformation property identifies $\mathcal{P}$, from the point of view of the representations of $\Gamma$, as $\mathcal{Q} \otimes \operatorname{End}(R)$. The space $\mathcal{P}$ can be given a quaternionic structure, representing its elements as "quaternions of matrices":

$$
p \in \mathcal{P}=\left(\begin{array}{cc}
A & i B^{\dagger} \\
i B & A^{\dagger}
\end{array}\right) \quad A, B \in \operatorname{End}(R)
$$

The space $\mathcal{S}$ is the subspace of $\Gamma$-invariant elements in $\mathcal{P}$ :

$$
\mathcal{S} \stackrel{\text { def }}{=}\{p \in \mathcal{P} / \forall \gamma \in \Gamma, \gamma \cdot p=p\}
$$

Explicitly the invariance condition reads:

$$
\left(\begin{array}{cc}
u_{\gamma} & i \bar{v}_{\gamma} \\
i v_{\gamma} & \bar{u}_{\gamma}
\end{array}\right)\left(\begin{array}{l}
A \\
B
\end{array}\right)=\left(\begin{array}{l}
R\left(\gamma^{-1}\right) A R(\gamma) \\
R\left(\gamma^{-1}\right) B R(\gamma)
\end{array}\right)
$$

The space $\mathcal{S}$ is elegantly described for all $\Gamma$ 's using the associated Dynkin diagram.

A two-vector of matrices can be thought of also as a matrix of two-vectors: that is, $\mathcal{P}=\mathcal{Q} \otimes \operatorname{Hom}(R, R)=\operatorname{Hom}(R, \mathcal{Q} \otimes R)$. Decomposing into irreducible representations the regular representation, $R=\bigoplus_{\nu=0}^{r} n_{\mu} D_{\mu}$, using eq.(23) and the Schur's lemma, one gets

$$
\mathcal{S}=\bigoplus_{\mu, \nu} A_{\mu, \nu} \operatorname{Hom}\left(\mathbf{C}^{n_{\mu}}, \mathbf{C}^{n_{\nu}}\right)
$$

The dimensions of the irrepses, $n_{\mu}$ are expressed in Fig.s (3,.3). From eq. (55) the real dimension of $\mathcal{S}$ follows immediately: $\operatorname{dim} \mathcal{S}=\sum_{\mu, \nu} 2 A_{\mu \nu} n_{\mu} n_{\nu}$ implies, recalling that $A=21-\bar{c}$ [see eq.(23)] and that for the extended Cartan matrix $\bar{c} n=0$, that

$$
\operatorname{dim} \mathcal{S}=4 \sum_{\mu} n_{\mu}^{2}=4|\Gamma|
$$

The quaternionic structure of $\mathcal{S}$ can be seen by simply writing its elements as in eq.(52) with $A, B$ satisfying the invariance condition eq.(54). Then the HyperKähler forms and the metric are described by $\Theta=\operatorname{Tr}(d \bar{m} \wedge m)$ and $d s^{2} \mathbf{1}=\operatorname{Tr}(d \bar{m} \otimes d m)$. The trace is taken over the matrices belonging to $\operatorname{End}(R)$ in each entry of the quaternion.

Example The space $\mathcal{S}$ can be easily described when $\Gamma$ is the cyclic group $A_{k-1}$. The order of $A_{k-1}$ is $k$; the abstract multiplication table is that of $\mathbf{Z}_{k}$. We can immediately read off from it the matrices of the regular representation; of course, it is sufficient to consider the representative of the first element $e_{1}$, as $R\left(e_{j}\right)=\left(R\left(e_{1}\right)\right)^{j}$. One has

$$
R\left(e_{1}\right)=\left(\begin{array}{ccccc}
0 & 0 & \cdots & 0 & 1 \\
1 & 0 & \cdots & 0 & 0 \\
0 & 1 & \cdots & 0 & 0 \\
\vdots & \vdots & \ddots & \vdots & \vdots \\
0 & 0 & \cdots & 1 & 0
\end{array}\right)
$$


Actually, the invariance condition eq.(54) is best solved by changing basis so as to diagonalize the regular representation, realizing explicitly its decomposition in terms of the $k$ unidimensional irrepses. Let $\nu=e^{\frac{2 \pi i}{k}}$, so that $\nu^{k}=1$. The wanted change of basis is performed by the matrix $S_{i j}=\frac{\nu^{-i j}}{\sqrt{k}}$, such that $S_{i j}^{-1}=\frac{\nu^{i j}}{\sqrt{k}}=S_{i j}^{\dagger}$. In the new basis $R\left(e_{1}\right)=\operatorname{diag}\left(1, \nu, \nu^{2}, \ldots, \nu^{k-1}\right)$, and so

$$
R\left(e_{j}\right)=\operatorname{diag}\left(1, \nu^{j}, \nu^{2 j}, \ldots, \nu^{(k-1) j}\right) .
$$

Eq.(58) displays on the diagonal the representatives of $e_{j}$ in the unidimensional irrepses.

The explicit solution of eq. (54) is given in the above basis by

$$
A=\left(\begin{array}{ccccc}
0 & u_{0} & 0 & \cdots & 0 \\
0 & 0 & u_{1} & \cdots & 0 \\
\vdots & \vdots & \vdots & \ddots & \vdots \\
\vdots & \vdots & \vdots & & u_{k-2} \\
u_{k-1} & 0 & 0 & \cdots & 0
\end{array}\right) \quad ; \quad B=\left(\begin{array}{ccccc}
0 & 0 & \cdots & \cdots & v_{0} \\
v_{1} & 0 & \cdots & \cdots & 0 \\
0 & v_{2} & \cdots & \cdots & 0 \\
\vdots & \vdots & \ddots & & \vdots \\
0 & 0 & \cdots & v_{k-1} & 0
\end{array}\right)
$$

We see that these matrices are parametrized in terms of $2 k$ complex, i.e. $4 k=\left|A_{k-1}\right|$ real parameters.

In the $D_{k+2}$ case, where the regular representation is $4 k$-dimensional, choosing appropriately a basis, one can solve analogously eq.(54); the explicit expressions are somehow space-consuming, so we don't write them. The essential point is that the matrices $A$ and $B$ no longer correspond to two distinct set of parameters, the group being non-abelian.

Consider the action of $S U(|\Gamma|)$ on $\mathcal{P}$ given, using the quaternionic notation for the elements of $\mathcal{P}$, by

$$
\forall g \in S U(|\Gamma|), g:\left(\begin{array}{cc}
A & i B^{\dagger} \\
i B & A^{\dagger}
\end{array}\right) \longmapsto\left(\begin{array}{cc}
g A g^{-1} & i g B^{\dagger} g^{-1} \\
i g B g^{-1} & g A^{\dagger} g^{-1}
\end{array}\right)
$$

It is easy to see that this action is a triholomorphic isometry of $\mathcal{P}$ : $d s^{2}$ and $\Theta$ are invariant. Let $F$ be the subgroup of $S U(|\Gamma|)$ which commutes with the action of $\Gamma$ on $\mathcal{P}$, with action described in eq.(51). Then the action of $F$ descends to $\mathcal{S} \subset \mathcal{P}$ to give a triholomorphic isometry: the metric and HyperKähler forms on $\mathcal{S}$ are just the restriction of those on $\mathcal{P}$. It is therefore possible to take the HyperKähler quotient of $\mathcal{S}$ with respect to $F$.

Let $\left\{f_{A}\right\}$ be a basis of generators for $\mathcal{F}$, the Lie algebra of $F$. Under the infinitesimal action of $f=\mathbf{1}+\lambda^{A} f_{A} \in F$, the variation of $m \in \mathcal{S}$ is $\delta m=\lambda^{A} \delta_{A} m$, with

$$
\delta_{A} m=\left(\begin{array}{cc}
{\left[f_{A}, A\right]} & i\left[f_{A}, B^{\dagger}\right] \\
i\left[f_{A}, B\right] & {\left[f_{A}, A^{\dagger}\right]}
\end{array}\right)
$$

The components of the momentum map (see (50)) are then given by

$$
\mu_{A}=\operatorname{Tr}\left(\bar{m} \delta_{A} m\right) \stackrel{\text { def }}{=} \operatorname{Tr}\left(\begin{array}{ll}
f_{A} \mu_{3}(m) & f_{A} \mu_{-}(m) \\
f_{A} \mu_{+}(m) & f_{A} \mu_{3}(m)
\end{array}\right)
$$


so that the real and holomorphic maps $\mu_{3}: \mathcal{S} \rightarrow \mathcal{F}^{*}$ and $\mu_{+}: \mathcal{S} \rightarrow \mathbf{C} \times \mathcal{F}^{*}$ can be represented as matrix-valued maps: ㅇ

$$
\begin{aligned}
\mu_{3}(m) & =-i\left(\left[A, A^{\dagger}\right]+\left[B, B^{\dagger}\right]\right) \\
\mu_{+}(m) & =([A, B]) .
\end{aligned}
$$

Let $\mathcal{Z}$ be the dual of the centre of $\mathcal{F}$. In correspondence of a level $\zeta=\left\{\zeta^{3}, \zeta^{+}\right\} \in \mathbf{R}^{3} \otimes \mathcal{Z}$ we can form the HyperKähler quotient $\mathcal{M}_{\zeta} \stackrel{\text { def }}{=} \mu^{-1} / F$. Varying $\zeta$ and $\Gamma$ every $A L E$ space can be obtained as $\mathcal{M}_{\zeta}$.

First of all, it is not difficult to check that $\mathcal{M}_{\zeta}$ is four-dimensional. As for the space $\mathcal{S}$, there is a nice characterization of the group $F$ in terms of the extended Dynkin diagram associated with $\Gamma$ :

$$
F=\bigotimes_{\mu} U\left(n_{\mu}\right)
$$

One must however set the determinant of the elements to be one, since $F \subset S U(|\Gamma|)$. $F$ has a $U\left(n_{\mu}\right)$ factor for each dot of the diagram, $n_{\mu}$ being associated to the dot as in Fig.s 3, 3]. F acts on the various "components" of $\mathcal{S}$ [which are in correspondence with the edges of the diagram, see eq.(55)] as dictated by the structure diagram. From eq. (64) is immediate to derive that $\operatorname{dim} F=\sum_{\mu} n_{\mu}^{2}-1=|\Gamma|-1$. It follows that

$$
\operatorname{dim} \mathcal{M}_{\zeta}=\operatorname{dim} \mathcal{S}-4 \operatorname{dim} F=4|\Gamma|-4(|\Gamma|-1)=4
$$

Example The structure of $F$ and the momentum map for its action are very simply worked out in the $A_{k-1}$ case. An element $f$ of $F$ must commute with the action of $A_{k-1}$ on $\mathcal{P}$, eq.(51), where the two-dimensional representation in the l.h.s. is given in eq.(24). Then $f$ must have the form

$$
f=\operatorname{diag}\left(e^{i \varphi_{0}}, e^{i \varphi_{1}}, \ldots, e^{i \varphi_{k-1}}\right) ; \sum \varphi_{i}=0
$$

Thus $\mathcal{F}$ is just the algebra of diagonal traceless $k$-dimensional matrices, which is $k-1$ dimensional. Choose a basis of generators for $\mathcal{F}$, for instance $f_{1}=\operatorname{diag}(1,-1, \ldots), f_{2}=$ $\operatorname{diag}(1,0,-1, \ldots), \ldots, f_{k-1}=\operatorname{diag}(1,0, \ldots,-1)$. From eq. (63) one gets directly the components of the momentum map:

$$
\begin{aligned}
\mu_{3, A} & =\left|u^{0}\right|^{2}-\left|v_{0}\right|^{2}-\left|u^{k-1}\right|^{2}-\left|v_{k-1}\right|^{2}-\left|u^{A}\right|^{2}-\left|v_{A}\right|^{2}+\left|u^{A-1}\right|^{2}-\left|v_{A-1}\right|^{2} \\
\mu_{+, A} & =u^{0} v_{o}-u^{k-1} v_{k-1}-u^{A} v_{A}+u^{A-1} v_{A-1} .
\end{aligned}
$$

In order for $\mathcal{M}_{\zeta}$ to be a manifold, it is necessary that $F$ act freely on $\mu^{-1}(\zeta)$. This happens or not depending on the value of $\zeta$. Again, a simple characterization of $\mathcal{Z}$ can

\footnotetext{
${ }^{2}$ It is easy to see that indeed the matrices $\left[A, A^{\dagger}\right]+\left[B, B^{\dagger}\right]$ and $[A, B]$ belong to the Lie algebra of traceless matrices $\mathcal{F}$; practically we identify $\mathcal{F}^{*}$ with $\mathcal{F}$ by means of the Killing metric.
} 
be given in terms of the simple Lie algebra $\mathcal{G}$ associated with $\Gamma$ [15. There exists an isomorphism between $\mathcal{Z}$ and the Cartan subalgebra $\mathcal{H}$ of $\mathcal{G}$. Thus we have

$$
\begin{aligned}
\operatorname{dim} \mathcal{Z}=\operatorname{dim} \mathcal{H} & =\operatorname{rank} \mathcal{G} \\
& =\# \text { of non trivial conj. classes in } \Gamma
\end{aligned}
$$

The space $\mathcal{M}_{\zeta}$ turns out to be singular when, under the above identification $\mathcal{Z} \sim \mathcal{H}$, any of the level components $\zeta^{i} \in \mathbf{R}^{3} \otimes \mathcal{Z}$ lies on the walls of a Weyl chamber. In particular, as the point $\zeta^{i}=0$ for all $i$ is identified with the origin in the root space, which lies of course on all the walls of the Weil chambers, the space $\mathcal{M}_{0}$ is singular. Without too much surprise we will see in a momentum that $\mathcal{M}_{0}$ corresponds to the orbifold limit $\mathbf{C}^{2} / \Gamma$ of a family of ALE manifolds with boundary at infinity $S^{3} / \Gamma$.

To see that this is general, choose the natural basis for the regular representation $R$, in which the basis vectors $e_{\delta}$ transform as in eq.(22). Define then the space $L \subset \mathcal{S}$ as follows:

$$
L=\left\{\left(\begin{array}{l}
C \\
D
\end{array}\right) \in \mathcal{S} / C, D \text { are diagonal in the basis }\left\{e_{\delta}\right\}\right\} .
$$

For every element $\gamma \in \Gamma$ there is a pair of numbers $\left(c_{\gamma}, d_{\gamma}\right)$ given by the corresponding entries of $C, D: C \cdot e_{\gamma}=c_{\gamma} e_{\gamma}, D \cdot e_{\gamma}=d_{\gamma} e_{\gamma}$. Applying the invariance condition eq.(54), which is valid since $L \subset \mathcal{S}$, it results that

$$
\left(\begin{array}{c}
c_{\gamma \cdot \delta} \\
d_{\gamma \cdot \delta}
\end{array}\right)=\left(\begin{array}{cc}
u_{\gamma} & i \bar{v}_{\gamma} \\
i v_{\gamma} & \bar{u}_{\gamma}
\end{array}\right)\left(\begin{array}{c}
c_{\delta} \\
d_{\delta}
\end{array}\right)
$$

We can identify $L$ with $\mathbf{C}^{2}$ associating for instance $(C, D) \in L \longmapsto\left(c_{0}, d_{0}\right) \in \mathbf{C}^{2}$. Indeed all the other pairs $\left(c_{\gamma}, d_{\gamma}\right)$ are determined in terms of eq. (70) once $\left(c_{0}, d_{0}\right)$ are given. By eq. (70) the action of $\Gamma$ on $L$ induces exactly the action of $\Gamma$ on $\mathbf{C}^{2}$ that we considered in (13, 14).

It is quite easy to show the following fundamental fact: each orbit of $F$ in $\mu^{-1}(0)$ meets $L$ in one orbit of $\Gamma$. Because of the above identification between $L$ and $\mathbf{C}^{2}$, this leads to prove that $X_{0}$ is isometric to $\mathbf{C}^{2} / \Gamma$.

In the spirit of the paper, instead of reviewing the proof of these statements (see [15]), we show explicitly the above facts in the case of the cyclic groups, giving a description which sheds some light on the deformed situation; that is we show in which way a nonzero level $\zeta^{+}$for the holomorphic momentum map puts $\mu^{-1}(\zeta)$ in correspondence with an hypersurface in $\mathbf{C}^{3}$ defined by a "potential" which is a deformation of the one describing the $\mathbf{C}^{2} / \Gamma$ situation, obtained for $\zeta^{+}=0$.

The case $\Gamma=A_{k-1}$

We can directly realize $\mathbf{C}^{2} / \Gamma$ as an affine algebraic surface in $\mathbf{C}^{3}$ (see eq. (27)) by expressing the coordinates $x, y$ and $z$ of $\mathbf{C}^{3}$ in terms of the matrices $(C, D) \in L$. The explicit parametrization of the matrices in $\mathcal{S}$ in the $A_{k-1}$ case (which was given in eq. (59) in the basis in which the regular representation $R$ is diagonal), can be conveniently 
rewritten in the "natural" basis $\left\{e_{\gamma}\right\}$ via the matrix $S^{-1}$ [see before eq.(58)]. The subset $L$ of diagonal matrices $(C, D)$ is given by

$$
C=c_{0} \operatorname{diag}\left(1, \nu, \nu^{2}, \ldots, \nu^{k-1}\right), \quad D=d_{0} \operatorname{diag}\left(1, \nu^{k-1}, \nu^{k-2}, \ldots, \nu\right)
$$

where $\nu=\mathrm{e}^{\frac{2 \pi i}{k}}$. This is nothing but the fact that $\mathbf{C}^{2} \sim L$. The set of pairs $\left(\begin{array}{c}\nu^{m} c_{0} \\ \nu^{k-m} d_{0}\end{array}\right)$, $m=0,1, \ldots, k-1$ is an orbit of $\Gamma$ in $\mathbf{C}^{2}$ and determines the corresponding orbit of $\Gamma$ in $L$. To describe $\mathbf{C}^{2} / A_{k-1}$ one needs to identify a suitable set of invariants $(x, y, z) \in \mathbf{C}^{3}$ such that $x y=z^{k}$, namely eq. (27). Our guess is

$$
x=\operatorname{det} C \quad ; \quad y=\operatorname{det} D, \quad ; \quad z=\frac{1}{k} \operatorname{Tr} C D .
$$

This guess will be confirmed in a moment by the study of the deformed surface.

We know that there is a one-to-one correspondence between the orbits of $F$ in $\mu^{-1}(0)$ and those of $\Gamma$ in $L$. Let us realize it explicitly. Choose the basis where $R$ is diagonal. Then $(A, B) \in \mathcal{S}$ has the form of eq. (59). Now, the relation $x y=z^{k}$ (eq. (27)) holds also true when, in eq. (72), the pair $(C, D) \in L$ is replaced by an element $(A, B) \in \mu^{-1}(0)$. To see this, let us describe the elements $(A, B) \in \mu^{-1}(0)$. We have to equate the right hand sides of eq. (63) to zero. We note that $[A, B]=0$ gives $v_{i}=\frac{u_{0} v_{0}}{v_{i}} \forall i$. Secondly, $\left[A, A^{\dagger}\right]+\left[B, B^{\dagger}\right]=0$ implies $\left|u_{i}\right|=\left|u_{j}\right|$ and $\left|v_{i}\right|=\left|v_{j}\right| \forall i, j$, i.e. $u_{j}=\left|u_{0}\right| \mathrm{e}^{i \phi_{j}}$ and $v_{j}=\left|v_{0}\right| \mathrm{e}^{i \psi_{j}}$. Finally, $[A, B]=0$ implies $\psi_{j}=\Phi-\phi_{j} \forall j$ for a certain phase $\Phi$. In this way, we have characterized $\mu^{-1}(0)$ and we immediately check that the pair $(A, B) \in \mu^{-1}(0)$ satisfies $x y=z^{k}$ if $x=\operatorname{det} A, y=\operatorname{det} B$ and $z=(1 / k) \operatorname{Tr} A B$. We are left with $k+3$ parameters (the $k$ phases $\phi_{j}, j=0,1, \ldots k-1$, plus the absolute values $\left|u_{0}\right|$ and $\left|v_{0}\right|$ and the phase $\Phi)$. Indeed $\operatorname{dim} \mu^{-1}(0)=\operatorname{dim} \mathcal{M}-3 \operatorname{dim} F=4|\Gamma|-3(|\Gamma|-1)=|\Gamma|+3$, where $|\Gamma|=\operatorname{dim} \Gamma=k$.

Now we perform the quotient of $\mu^{-1}(0)$ with respect to $F$. Given a set of phases $f_{i}$ such that $\sum_{i=0}^{k-1} f_{i}=0 \bmod 2 \pi$ and given $f=\operatorname{diag}\left(\mathrm{e}^{i f_{0}}, \mathrm{e}^{i f_{1}}, \ldots, \mathrm{e}^{i f_{k-1}}\right) \in F$, the orbit of $F$ in $\mu^{-1}(0)$ passing through $\left(\begin{array}{l}A \\ B\end{array}\right)$ is given by $\left(\begin{array}{c}f A f^{-1} \\ f B f^{-1}\end{array}\right)$. Choosing $f_{j}=f_{0}+j \psi+\sum_{n=0}^{j-1} \phi_{n}$, $j=1, \ldots, k-1$, with $\psi=-\frac{1}{k} \sum_{n=0}^{k-1} \phi_{n}$, and $f_{0}$ determined by the condition $\sum_{i=0}^{k-1} f_{i}=$ $0 \bmod 2 \pi$, one has

$$
f A f^{-1}=a_{0}\left(\begin{array}{ccccc}
0 & 1 & 0 & \ldots & 0 \\
0 & 0 & 1 & \ldots & 0 \\
& \ldots & & \ldots & \\
0 & 0 & \ldots & 0 & 1 \\
1 & 0 & 0 & \ldots & 0
\end{array}\right), f B f^{-1}=b_{0}\left(\begin{array}{ccccc}
0 & 0 & \ldots & 0 & 1 \\
1 & 0 & 0 & \ldots & 0 \\
0 & 1 & 0 & \ldots & 0 \\
& \ldots & & \ldots & \\
0 & \ldots & 0 & 1 & 0
\end{array}\right)
$$

where $a_{0}=\left|u_{0}\right| \mathrm{e}^{i \psi}$ and $b_{0}=\left|v_{0}\right| \mathrm{e}^{i(\Phi-\psi)}$. Since the phases $\phi_{j}$ are determined modulo $2 \pi$, it follows that $\psi$ is determined modulo $\frac{2 \pi}{k}$. Thus we can say $\left(a_{0}, b_{0}\right) \in \mathbf{C}^{2} / \Gamma$. This is the one-to-one correspondence between $\mu^{-1}(0) / F$ and $\mathbf{C}^{2} / \Gamma$. 
We now derive the deformed relation between the invariants $x, y, z$. It fixes the correspondence between the resolution of singularity performed in the momentum map approach and the resolution performed on the hypersurface $x y=z^{k}$ in $C^{3}$. To this purpose, we focus on the holomorphic part of the momentum map, i.e. on the equation $[A, B]=\Lambda_{0}$, where $\Lambda_{0}=\operatorname{diag}\left(\lambda_{0}, \lambda_{1}, \lambda_{2}, \ldots, \lambda_{k-1}\right) \in \mathcal{Z} \otimes \mathbf{C}^{2}$ with $\lambda_{0}=-\sum_{i=1}^{k-1} \lambda_{i}$. Recall expression (59) for the matrices $A$ and $B$. Calling $a_{i}=u_{i} v_{i},[A, B]=\Lambda_{0}$ implies that $a_{i}=a_{0}+\lambda_{i}$ for $i=1, \ldots, k-1$. Now, let $\Lambda=\operatorname{diag}\left(\lambda_{1}, \lambda_{2}, \ldots, \lambda_{k-1}\right)$. We have

$$
x y=\operatorname{det} A \operatorname{det} B=a_{0} \Pi_{i=1}^{k-1}\left(a_{0}+\lambda_{i}\right)=a_{0}^{k} \operatorname{det}\left(1+\frac{1}{a} \Lambda\right)=\sum_{i=0}^{k-1} a_{0}^{k-i} S_{i}(\Lambda) .
$$

The $S_{i}(\Lambda)$ are the symmetric polynomials in the eigenvalues of $\Lambda$. They are defined by the relation $\operatorname{det}(1+\Lambda)=\sum_{i=0}^{k-1} S_{i}(\Lambda)$ and are given by $S_{i}(\Lambda)=\sum_{j_{1}<j_{2}<\cdots<j_{i}} \lambda_{j_{1}} \lambda_{j_{2}} \cdots \lambda_{j_{i}}$. In particular, $S_{0}=1$ and $S_{1}=\sum_{i=1}^{k-1} \lambda_{i}$. Define $S_{k}(\Lambda)=0$, so that $x y=\sum_{i=0}^{k} a_{0}^{k-i} S_{i}(\Lambda)$, and note that

$$
z=\frac{1}{k} \operatorname{Tr} A B=a_{0}+\frac{1}{k} S_{1}(\Lambda) .
$$

Then the desired deformed relation between $x, y$ and $z$ is obtained by substituting $a_{0}=$ $z-\frac{1}{k} S_{1}$ in (74), thus obtaining

$$
\begin{aligned}
& x y=\sum_{m=0}^{k} \sum_{n=0}^{k-m}\left(\begin{array}{c}
k-m \\
n
\end{array}\right)\left(-\frac{1}{k} S_{1}\right)^{k-m-n} S_{m} z^{n}=\sum_{n=0}^{k} t_{n} z^{n} . \\
& \Longrightarrow \quad t_{n}=\sum_{m=0}^{k-n}\left(\begin{array}{c}
k-n \\
m
\end{array}\right)\left(-\frac{1}{k} S_{1}\right)^{k-m-n} S_{n} .
\end{aligned}
$$

Notice in particular that $t_{k}=1$ and $t_{k-1}=0$, i.e. $x y=z^{k}+\sum_{n=0}^{k-2} t_{n} z^{n}$, which means that the deformation proportional to $z^{k-1}$ is absent. This establishes a clear correspondence between the momentum map construction and the polynomial ring $\frac{C[x, y, z]}{\partial W}$ where $W(x, y, z)=x y-z^{k}$ (compare with eq. (28)). Moreover, note that we have only used one of the momentum map equations, namely $[A, B]=\Lambda_{0}$. The equation $\left[A, A^{\dagger}\right]+\left[B, B^{\dagger}\right]=\Sigma$ has been completely ignored. This means that the deformation of the complex structure is described by the parameters $\Lambda$, while the parameters $\Sigma$ describe the deformation of the complex structure.

The relation (77) can also be written in a simple factorized form, namely

$$
x y=\Pi_{i=0}^{k-1}\left(z-\mu_{i}\right),
$$

where

$$
\begin{aligned}
\mu_{i} & =\frac{1}{k}\left(\lambda_{1}+\lambda_{2}+\cdots+\lambda_{i-1}-2 \lambda_{i}+\lambda_{i+1}+\cdots+\lambda_{k}\right), \quad i=1, \ldots, k-1 \\
\mu_{0} & =-\sum_{i=1}^{k} \mu_{i}=\frac{1}{k} S_{1} .
\end{aligned}
$$


The case $\Gamma=D_{k+2}$

The case $\Gamma=D_{k+2}$ cannot be treated with the algebraic simplicity of the previous one. Nevertheless, we can give an ansatz for the expressions of $x, y$ and $z$ in terms of the matrices $(A, B) \in \mu^{-1}(\zeta) / F$. This ansatz surely works for the undeformed case $\zeta=0$, because it can be checked via the correspondence between $\mu^{-1}(0) / F$ and $\mathbf{C}^{2} / \Gamma$ that permits to manage with diagonal matrices $C$ and $D$ instead of $A$ and $B$. Let (compare with (38))

$$
\begin{aligned}
x & =\frac{i}{8 k} \operatorname{Tr}\left[A^{2 k+1} B-(-1)^{k} A B^{2 k+1}\right], \\
y & =\frac{i}{8 k} \operatorname{Tr}\left[A^{2 k}+(-1)^{k} B^{2 k}\right] \\
z & =-\frac{1}{16 k} \operatorname{Tr}\{A, B\}^{2} .
\end{aligned}
$$

In the undeformed case $\zeta=0$, the relation $[A, B]=0$ shows that one cannot fix $z$ unambiguously, because expressions proportional to $\operatorname{Tr} A^{2} B^{2}$ or $\operatorname{Tr}(A B)^{2}$ are equally allowed. To resolve the ambiguity, we have worked out the deformation in the simplest case, namely $k=1$. $A$ and $B$ are $4 \times 4$ matrices. In a suitable basis they have the form

$$
A=\left(\begin{array}{llll}
0 & a & 0 & b \\
c & 0 & d & 0 \\
0 & e & 0 & f \\
g & 0 & h & 0
\end{array}\right), \quad B=i\left(\begin{array}{cccc}
0 & -f & 0 & e \\
-h & 0 & -g & 0 \\
0 & -b & 0 & a \\
d & 0 & c & 0
\end{array}\right) .
$$

In our case the explicit form of the momentum map equation is $\mu_{+}(m) \equiv[A, B]=\Lambda$ [see eq. [63)], that we write as

$$
[A, B]=i\left(\begin{array}{cccc}
l_{1} & 0 & l_{2} & 0 \\
0 & -l_{1} & 0 & l_{3} \\
l_{2} & 0 & l_{1} & 0 \\
0 & -l_{3} & 0 & -l_{1}
\end{array}\right)
$$

Then we have

$$
x^{2}+y^{2} z+z^{2}+t_{1}+t_{2} y+t_{3} y^{2}+t_{4} z=0,
$$

where

$$
\begin{aligned}
t_{1} & =-\frac{1}{16}\left[l_{2}^{2} l_{3}^{2}-\frac{1}{4}\left(2 l_{1}^{2}-l_{2}^{2}+l_{3}^{2}\right)\right], \\
t_{2} & =-\frac{i}{4} l_{1} l_{2} l_{3}, \\
t_{3} & =\frac{1}{8}\left(l_{2}^{2}-l_{3}^{2}\right), \\
t_{4} & =-\frac{1}{4} l_{1}^{2} .
\end{aligned}
$$

Note the presence of both $y^{2}$ and $z$ in the deformed relation, although one vanishing relation of the chiral ring says that they are proportional. One can make the $y^{2}$-term disappear by simply performing a $l$-dependent translation of $z$. 


\section{The orbifold $\mathrm{C}^{2} / \Gamma$ conformal field theory}

Now we address the problem of constructing the $(4,4)$ conformal field theory associated with an ALE instanton. This can be explicitly done in the orbifold limit $\mathcal{M}_{0}=\mathbf{C}^{2} / \Gamma$, corresponding to $\left\{\zeta^{i}=0\right\}$. The $(4,4)$ theories associated with the smooth manifolds $\mathcal{M}_{\zeta}$ can be obtained from the orbifold theory by perturbing it with the moduli operators associated with the elements of the ring $\mathbf{C}[x, y, z] / \partial W$.

After briefly reviewing the structure of the $N=4$ algebra and its possible representations at $c=6$, we give a description of the $(4,4)$ orbifold theory in terms of primary fields and OPEs. Next we consider the structure of the corresponding partition function, and we analyse it in terms of $N=4$ characters.

The $N=4$ algebra is described in terms of OPEs as follows:

$$
\begin{aligned}
T(z) T(w) & =\frac{c}{2} \frac{1}{(z-w)^{4}}+\frac{2 T(w)}{(z-w)^{2}}+\frac{\partial T(w)}{z-w} \\
T(z) \mathcal{G}^{a}(w) & =\frac{3}{2} \frac{\mathcal{G}^{a}(w)}{(z-w)^{2}}+\frac{\partial \mathcal{G}^{a}}{z-w} ; T(z) \overline{\mathcal{G}}^{a}(w)=\frac{3}{2} \frac{\overline{\mathcal{G}}^{a}(w)}{(z-w)^{2}}+\frac{\partial \overline{\mathcal{G}}^{a}}{z-w} \\
T(z) A^{i}(w) & =\frac{A^{i}(w)}{(z-w)^{2}}+\frac{\partial A^{i}(w)}{z-w} \\
A^{i}(z) \mathcal{G}^{a}(w) & =\frac{1}{2} \frac{\mathcal{G}^{b}(w)\left(\sigma^{i}\right)^{b a}}{z-w} ; A^{i}(z) \overline{\mathcal{G}}^{a}(w)=-\frac{1}{2} \frac{\overline{\mathcal{G}}^{b}(w)\left(\sigma^{i}\right)^{a b}}{z-w} \\
\mathcal{G}^{a}(z) \overline{\mathcal{G}}^{b}(w) & =\frac{2}{3} c \frac{\delta^{a b}}{(z-w)^{3}}+\frac{4\left(\sigma_{i}^{*}\right)^{a b} A^{i}(w)}{(z-w)^{2}}+2 \frac{\delta^{a b} T(w)+\partial A^{i}(w)\left(\sigma_{i}^{*}\right)^{a b}}{z-w} \\
A^{i}(z) A^{j}(w) & =\frac{1}{12} c \frac{\delta^{i j}}{(z-w)^{2}}+i \varepsilon^{i j k} \frac{A^{(k)}(w)}{z-w} .
\end{aligned}
$$

Note that in the above OPEs and in all the following ones the equality sign means equality up to regular terms. In general, the central charge $c$ is an integer multiple of 6 in a unitary theory, but we shall only be interested in the case $c=6$.

To discuss the structure of the representations [30], using the highest-weight method, it is convenient to rewrite the $N=4$ algebra (85) in terms of modes:

$$
\begin{aligned}
& {\left[L_{m}, L_{n}\right]=(m-n) L_{m+n}+\frac{1}{2} k m\left(m^{2}-1\right) \delta_{m+n, 0}} \\
& {\left[L_{m}, \mathcal{G}_{r}^{a}\right]=\left(\frac{1}{2} m-r\right) \mathcal{G}_{m+r}^{a}, \quad\left[L_{m}, \overline{\mathcal{G}}_{s}^{a}\right]=\left(\frac{1}{2} m-s\right) \overline{\mathcal{G}}_{m+s}^{a}} \\
& {\left[L_{m}, A_{n}^{i}\right]=-n A_{m+n}^{i}} \\
& {\left[A_{m}^{i}, \mathcal{G}_{r}^{a}\right]=\frac{1}{2} \sigma_{b a}^{i} \mathcal{G}_{m+r}^{b}, \quad\left[A_{m}^{i}, \overline{\mathcal{G}}_{s}^{a}\right]=-\frac{1}{2} \sigma_{b a}^{i *} \overline{\mathcal{G}}_{m+s}^{b}} \\
& \left\{\mathcal{G}_{r}^{a}, \mathcal{G}_{s}^{b}\right\}=\left\{\overline{\mathcal{G}}_{r}^{a}, \overline{\mathcal{G}}_{s}^{b}\right\}=0 \\
& \left\{\mathcal{G}_{r}^{a}, \overline{\mathcal{G}}_{s}^{b}\right\}=2 \delta^{a b} L_{r+s}-2(r-s) \sigma_{a b}^{i} A_{r+s}^{i}+\frac{1}{2} k\left(4 r^{2}-1\right) \delta_{r+s, 0} \delta_{a b}
\end{aligned}
$$




$$
\left[A_{m}^{i}, A_{n}^{j}\right]=i \epsilon_{i j k} A_{m+n}^{k}+\frac{1}{2} k m \delta_{m+n, 0} \delta^{i j}
$$

where indices $r, s$ take integral values in the Ramond sector $(\mathrm{R})$ and half-integer values in the Neveu-Schwarz (NS) sector. The value of the central charge being $c=6 k$, only the case $k=1$ is relevant to our discussion, as already stated. Furthermore we can restrict ourselves to the NS sector, since the Ramond sector can be reached by spectral-flow.

The highest-weight states of the $\mathrm{N}=4$ algebra are defined by the conditions:

$$
\begin{aligned}
& L_{n}|h, l\rangle=\mathcal{G}_{r}^{a}|h, l\rangle=\overline{\mathcal{G}}_{s}^{b}|h, l\rangle=A_{n}^{i}|h, l\rangle=0, \quad n \geq 1, \quad r, s \geq \frac{1}{2} \\
& A_{0}^{+}|h, l\rangle=0 \\
& L_{0}|h, l\rangle=h|h, l\rangle, \quad T_{0}^{3}|h, l\rangle=l|h, l\rangle .
\end{aligned}
$$

Unitarity puts the restriction $h \geq l$. There exist two classes of unitary representations of the $\mathrm{N}=4$ algebra: the long representations

$$
h>l, \quad l=0, \frac{1}{2}, \ldots, \frac{1}{2}(k-1)
$$

and the short ones

$$
h=l, \quad l=0, \frac{1}{2}, \ldots, \frac{1}{2} .
$$

The short representations exist when $h$ saturates the unitary bound $h=l$ and the long representations decompose in short ones in the limit in which $h$ reaches $l$. The unitary bound $h \geq l$ is the $N=4$ transcription of the analogous $N=2$ bound $h \geq|q| / 2$. The short representations are in fact defined to obey the condition

$$
\mathcal{G}_{-\frac{1}{2}}^{2}|h, l\rangle=\overline{\mathcal{G}}_{-\frac{1}{2}}^{1}|h, l\rangle=0
$$

which is in fact equivalent to $h=l$ by commutation relations. In other terms, $|h, h\rangle$ can be constructed using the chiral fields of the corresponding $\mathrm{N}=2$ algebra. Starting from the highest-weight state we can try to close an $\mathrm{N}=4$ superconformal representation by repeated application of the generators. The result can be conveniently retrieved and expressed in terms of OPEs, as follows.

Note that for $c=6$ there are only two type of short representations: the $|0,0\rangle$ case, corresponding only to the identity in a unitary conformal theory, and the $\left|\frac{1}{2}, \frac{1}{2}\right\rangle$ case, the only non trivial one.

Using the notation introduced in section 1, the multiplet of a short representation is

$$
\left(\Psi^{a}\left[\begin{array}{l}
1 / 2 \\
1 / 2
\end{array}\right], \Phi\left[\begin{array}{l}
1 \\
0
\end{array}\right], \Pi\left[\begin{array}{l}
1 \\
0
\end{array}\right]\right) \text {. }
$$

It is characterized by the following OPEs with the supercurrents:

$$
\begin{aligned}
\mathcal{G}^{a}(z) \Psi^{b}(w) & =\delta^{a b} \frac{\Phi(w)}{z-w} \quad ; \quad \overline{\mathcal{G}}^{a}(z) \Psi^{b}(w)=\epsilon^{a b} \frac{\Pi(w)}{z-w} \\
\mathcal{G}^{a}(z) \Pi(w) & =-2 \varepsilon^{a b} \partial_{w}\left(\frac{\Psi^{a}}{z-w}\right) ; \quad \overline{\mathcal{G}}^{a}(z) \Phi(w)=2 \partial_{w}\left(\frac{\Psi^{a}}{z-w}\right) .
\end{aligned}
$$


The first two OPEs define the short representations, while the other OPEs are consequences of the first two OPEs.

This can be seen by using two Jacobi-like identities, that can be written as

$$
\begin{aligned}
& \oint \frac{z^{m} d z}{2 \pi i} \oint \frac{\zeta^{n} d \zeta}{2 \pi i} D^{ \pm}(z) \cdot(G(\zeta) \cdot \mathcal{O}(w))= \\
& =\oint \frac{z^{m} d z}{2 \pi i} \oint \frac{\zeta^{n} d \zeta}{2 \pi i}\left(\left(D^{ \pm}(z) \cdot G(\zeta)\right) \cdot O(w) \pm G(\zeta) \cdot\left(D^{ \pm}(z) \cdot \mathcal{O}(w)\right)\right)
\end{aligned}
$$

$\forall m, \forall n$ and $\forall \mathcal{O}(w)$, with $D^{ \pm}(z)$ bosonic (resp. fermionic) and $G(\zeta)$ fermionic. The dot means OPE expansion. We specify the order in which the OPE's are to be performed instead of specifying the equivalent information on the integration contours. One can use the cases $m, n=0,1$ to extract information about simple and double poles. Explicitly, set

$$
\begin{aligned}
\varepsilon^{a b} \Phi(w) & =\oint \frac{d \zeta}{2 \pi i} \mathcal{G}^{a}(\zeta) \Psi^{b}(w), \\
\delta^{a b} \Pi(w) & =\oint \frac{d \zeta}{2 \pi i} \overline{\mathcal{G}}^{a}(\zeta) \Psi^{b}(w) .
\end{aligned}
$$

With $\mathcal{O}(w)=\Psi^{b}(w)$ and $G(\zeta)=\mathcal{G}^{a}(\zeta)$ or $\overline{\mathcal{G}}^{a}(\zeta)$, the identities (93) can be used alternatively to check the conformal weight of $\Phi$ and $\Pi$ (by choosing $D^{+}(z)=T(z)$ ), to check the $S U(2)$ representation (with $D^{+}(z)=A^{i}(z)$ ) and finally to check the other OPEs of eq. (92) $\left(D^{-}(z)=\mathcal{G}^{a}(\zeta)\right.$ or $\left.\overline{\mathcal{G}}^{a}(\zeta)\right)$.

The multiplet of a long representations is instead (see Fig. 5).

$$
\left(\Omega\left[\begin{array}{l}
h \\
0
\end{array}\right], \Phi^{a}\left[\begin{array}{c}
h+1 / 2 \\
1 / 2
\end{array}\right], \bar{\Phi}^{a}\left[\begin{array}{c}
h+1 / 2 \\
1 / 2
\end{array}\right], \Gamma\left[\begin{array}{c}
h+1 \\
0
\end{array}\right], \bar{\Gamma}\left[\begin{array}{c}
h+1 \\
0
\end{array}\right], \Sigma\left[\begin{array}{c}
h+1 \\
0
\end{array}\right]\right),
$$

with $h>0$. Its OPEs with the supercurrents are

$$
\begin{aligned}
\mathcal{G}^{a}(z) \Omega(w) & =\frac{\Phi^{a}(w)}{z-w} ; \quad \overline{\mathcal{G}}^{a}(z) \Omega(w)=\frac{\bar{\Phi}^{a}(w)}{z-w} \\
\mathcal{G}^{a}(z) \Phi^{b}(w) & =\varepsilon^{a b} \frac{\Gamma(w)}{z-w} ; \quad \overline{\mathcal{G}}^{a}(z) \bar{\Phi}^{b}(w)=\varepsilon^{a b} \frac{\bar{\Gamma}(w)}{z-w} \\
\overline{\mathcal{G}}^{a}(z) \Phi^{b} & =2 h \delta^{a b} \frac{\Omega(w)}{(z-w)^{2}}+\delta^{a b} \frac{\partial \Omega(w)}{z-w}+\frac{1}{2} \delta^{a b} \frac{\Sigma(w)}{z-w}-8 h \frac{\left(\sigma_{i}\right)^{a b} A^{i}(w) \Omega(w)}{z-w} \\
\mathcal{G}^{a}(z) \bar{\Phi}^{b} & =2 h \delta^{a b} \frac{\Omega(w)}{(z-w)^{2}}+\delta^{a b} \frac{\partial \Omega(w)}{z-w}-\frac{1}{2} \delta^{a b} \frac{\Sigma(w)}{z-w}+8 h \frac{\left(\sigma_{i}^{*}\right)^{a b} A^{i}(w) \Omega(w)}{z-w} \\
\mathcal{G}^{a}(z) \bar{\Gamma}(w) & =2(h+1) \varepsilon^{a b} \frac{\bar{\Phi}^{b}}{(z-w)^{2}}+2 \varepsilon^{a b} \frac{\partial \bar{\Phi}^{b}}{z-w}+2 h \varepsilon^{a b} \frac{\Delta \bar{\Phi}^{b}}{z-w} \\
\overline{\mathcal{G}}^{a}(z) \Gamma(w) & =2(h+1) \varepsilon^{a b} \frac{\Phi^{b}}{(z-w)^{2}}+2 \varepsilon^{a b} \frac{\partial \Phi^{b}}{z-w}+2 h \varepsilon^{a b} \frac{\Delta \Phi^{b}}{z-w} \\
\mathcal{G}^{a}(z) \Sigma(w) & =2(h+1) \frac{\Phi^{a}}{(z-w)^{2}}+2 \frac{\partial \Phi^{a}}{z-w}+2 h \frac{\Delta \Phi^{a}}{z-w}
\end{aligned}
$$



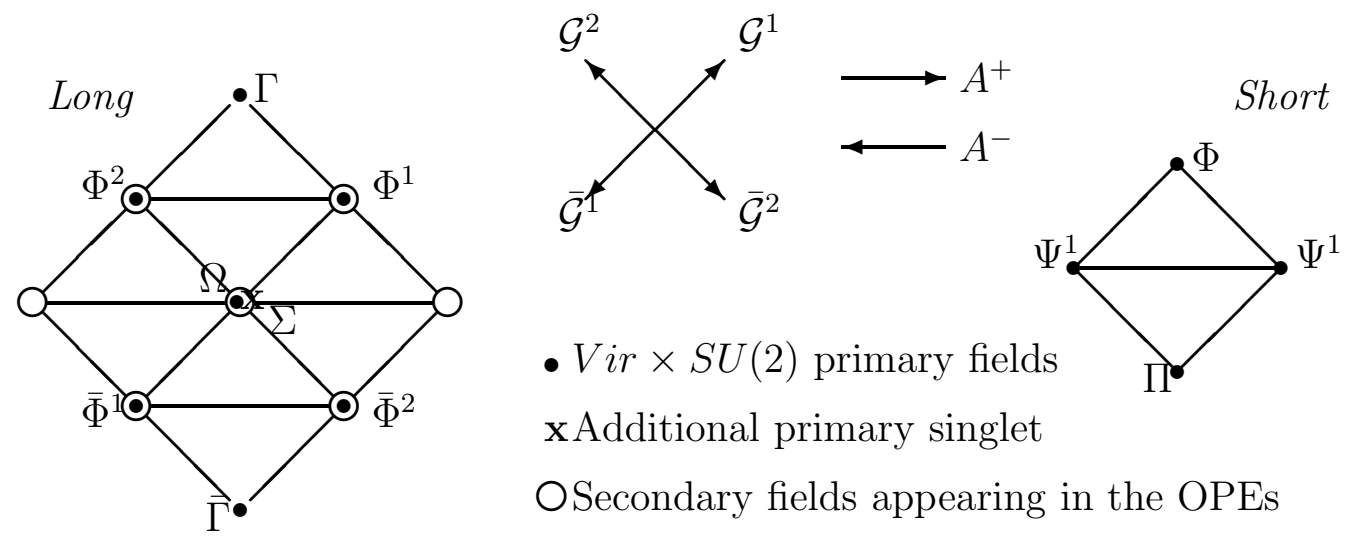

xAdditional primary singlet

OSecondary fields appearing in the OPEs

Figure 4: Long and short representations

$$
\overline{\mathcal{G}}^{a}(z) \Sigma(w)=-2(h+1) \frac{\bar{\Phi}^{a}}{(z-w)^{2}}-2 \frac{\partial \bar{\Phi}^{a}}{z-w}-2 h \frac{\Delta \bar{\Phi}^{a}}{z-w}
$$

where $\Delta \Phi^{a}(w)=\lim _{w^{\prime} \rightarrow w}\left[4\left(\sigma_{i}\right)^{a b} A^{i}(w) \Phi^{b}\left(w^{\prime}\right)-2 \mathcal{G}^{a}(w) \Omega\left(w^{\prime}\right)\right]$. As in the case of the short representations, the first two OPEs are assumptions. They define the highest weight operator $\Omega$ of the long representation. All the other OPEs are consequences of the first two and the Jacobi identities (93) as in the massless case.

The structure of the above OPEs is well summarized in Figure 5 that can be seen also as describing the algebra closed on the representations by the zero-modes of the generators.

Orbifold Conformal Field Theory of $\mathbf{C}^{2} / \Gamma$

Now we consider the explicit construction of the orbifold conformal field theory $\mathbf{C}^{2} / \Gamma$, starting from the $(4,4)$ theory of $\mathbf{C}^{2}$. Let $X, \bar{X}$ and $Y, \bar{Y}$ be two complex bosonic fields, $\psi_{x}, \bar{\psi}_{x}$ and $\psi_{y}, \bar{\psi}_{y}$ two complex fermions. They are normalized according to

$$
\begin{aligned}
\partial X(z) \partial \bar{X}(w) & =-\frac{2}{(z-w)^{2}}, \\
\psi_{x}(z) \bar{\psi}_{x}(w) & =-\frac{2}{z-w} .
\end{aligned}
$$

The $\mathrm{N}=4$ superconformal algebra is realized by setting

$$
\begin{aligned}
& T(z)=-\frac{1}{2}(\partial X \partial \bar{X}+\partial Y \partial \bar{Y})+\frac{1}{4}\left(\bar{\psi}_{x} \partial \psi_{x}-\partial \bar{\psi}_{x} \psi_{x}+\bar{\psi}_{y} \partial \psi_{y}-\partial \bar{\psi}_{y} \psi_{y}\right), \\
& A^{i}(z)=\frac{1}{4}\left[\begin{array}{c}
i\left(\psi_{x} \psi_{y}+\bar{\psi}_{x} \bar{\psi}_{y}\right) \\
\bar{\psi}_{x} \bar{\psi}_{y}-\psi_{x} \psi_{y} \\
\psi_{x} \bar{\psi}_{x}+\psi_{y} \bar{\psi}_{y}
\end{array}\right], \\
& \mathcal{G}^{a}(z)=\frac{1}{\sqrt{2}}\left[\begin{array}{c}
\bar{\psi}_{x} \\
i \psi_{y}
\end{array}\right] \partial X+\frac{1}{\sqrt{2}}\left[\begin{array}{c}
\bar{\psi}_{y} \\
-i \psi_{x}
\end{array}\right] \partial Y, \\
& \overline{\mathcal{G}}^{a}(z)=\frac{1}{\sqrt{2}}\left[\begin{array}{c}
\psi_{x} \\
-i \bar{\psi}_{y}
\end{array}\right] \partial \bar{X}+\frac{1}{\sqrt{2}}\left[\begin{array}{c}
\psi_{y} \\
i \bar{\psi}_{x}
\end{array}\right] \partial \bar{Y} .
\end{aligned}
$$


The short representations can be easily obtained by looking at the doublets that appear in the supercurrents. We have

$$
\begin{gathered}
\left(\Psi^{a}=\frac{1}{\sqrt{2}}\left[\begin{array}{c}
\psi_{x} \\
-i \bar{\psi}_{y}
\end{array}\right], \Phi=-\partial X, \Pi=-i \partial \bar{Y}\right), \\
\left(\frac{1}{\sqrt{2}}\left[\begin{array}{c}
\psi_{y} \\
i \bar{\psi}_{x}
\end{array}\right],-\partial Y, i \partial \bar{X}\right) .
\end{gathered}
$$

These representations satisfy the OPEs (92).

The long representations are

$$
\begin{aligned}
\Omega= & \exp i\left(p_{x} X+\bar{p}_{x} \bar{X}+p_{y} Y+\bar{p}_{y} \bar{Y}\right) \\
\Phi^{a}= & -i \sqrt{2}\left[\begin{array}{c}
\bar{p}_{x} \bar{\psi}_{x}+\bar{p}_{y} \bar{\psi}_{y} \\
i \bar{p}_{x} \psi_{y}-i \bar{p}_{y} \psi_{x}
\end{array}\right] \Omega, \\
\bar{\Phi}^{a}= & -i \sqrt{2}\left[\begin{array}{c}
p_{x} \psi_{x}+p_{y} \psi_{y} \\
-i p_{x} \bar{\psi}_{y}+i p_{y} \bar{\psi}_{x}
\end{array}\right] \Omega, \\
\Gamma= & 2\left[\bar{p}_{x} \partial Y-\bar{p}_{y} \partial X+i\left(\bar{p}_{x} \bar{\psi}_{x}+\bar{p}_{y} \bar{\psi}_{y}\right)\left(\bar{p}_{x} \psi_{y}-\bar{p}_{y} \psi_{x}\right)\right] \Omega, \\
\bar{\Gamma}= & 2\left[p_{y} \partial \bar{X}-p_{x} \partial \bar{Y}+i\left(p_{x} \psi_{x}+p_{y} \psi_{y}\right)\left(p_{y} \bar{\psi}_{x}-p_{x} \bar{\psi}_{y}\right)\right] \Omega, \\
\Sigma= & 2\left[i\left(p_{x} \partial X-\bar{p}_{x} \partial \bar{X}+p_{y} \partial Y-\bar{p}_{y} \partial \bar{Y}\right)-\left(\left|p_{x}\right|^{2}-\left|p_{y}\right|^{2}\right)\left(\bar{\psi}_{x} \psi_{x}-\bar{\psi}_{y} \psi_{y}\right)\right. \\
& \left.-2 \bar{p}_{x} p_{y} \bar{\psi}_{x} \psi_{y}+2 p_{x} \bar{p}_{y} \psi_{x} \bar{\psi}_{y}\right] \Omega .
\end{aligned}
$$

Now we turn to the study of the orbifold conformal field theory, and, after a brief review of the generalities on orbifold constructions [17], we will focus on the case $\Gamma=$ $A_{n-1}$.

The construction of the orbifold conformal field theory $\mathbf{C}^{2} / \Gamma$ begins with a Hilbert space projection onto $\Gamma$ invariant states. This projection can be represented in lagrangian form as the sum over contributions of fields twisted in temporal direction by all the elements of the group, i.e. $x(\sigma, \tau+2 \pi)=\gamma x(\sigma, \tau)$. $\mathrm{n}$ hamiltonian language the twisted boundary conditions correspond to insertion of the operator implementing $\gamma$ in the Hilbert space, as it wiil be explained with more details in section 6, and hence the sum $\sum_{\gamma \in \Gamma} g$ realizes the projection operator onto $\Gamma$ invariant states. To obtain a modular invariant theory we are forced to consider also twisted boundary conditions in the spatial direction, i.e. $x(\sigma+2 \pi, \tau)=\gamma x(\sigma, \tau)$; from the stringy point of view, these sectors correspond to the case in which the string is closed only modulo a transformation of the group $\Gamma$. One may think to have a different boundary condition for every element of the group; actually there is a boundary condition for each conjugacy class of the group, for, if the field obeys

$$
x(z+1)=\gamma x(z)
$$

it also obeys

$$
\eta x(z+1)=\left(\eta \gamma \eta^{-1}\right) \eta x(z)
$$

where $\eta$ is any other element of $\Gamma$. So the sectors twisted by $\eta \gamma \eta^{-1}$ are in fact all the same sector. 
We have to introduce "twist" operators which applied to the vacuum realize the change of sector in the Hilbert space, modifying the monodromy properties of the fields. Such situation recalls what happens for fermions, where we are explicitly able to construct spin fields which change the boundary conditions of the fermionic fields.

For the description of the monodromy properties of the fermions $\psi_{x}(z), \psi_{y}(z), \tilde{\psi}_{x}(\bar{z})$, $\tilde{\psi}_{y}(\bar{z})$, in the $A_{n-1}$ case, namely

$$
\begin{array}{ccc}
\psi_{x}\left(\mathrm{e}^{2 \pi i} z\right)=\mathrm{e}^{2 \pi i \frac{k}{n}} \psi_{x}(z), & \psi_{y}\left(\mathrm{e}^{2 \pi i} z\right)=\mathrm{e}^{2 \pi i \frac{n-k}{n}} \psi_{y}(z), \\
\tilde{\psi}_{x}\left(\mathrm{e}^{-2 \pi i} \bar{z}\right)=\mathrm{e}^{2 \pi i \frac{n-k}{n}} \tilde{\psi}_{x}(\bar{z}), & \tilde{\psi}_{y}\left(\mathrm{e}^{-2 \pi i} \bar{z}\right)=\mathrm{e}^{2 \pi i \frac{k}{n}} \tilde{\psi}_{y}(\bar{z}),
\end{array}
$$

we introduce the spin fields $s_{x}^{(k)}(z), s_{y}^{(k)}(z)$ and their world-sheet complex conjugates $\tilde{s}_{x}^{(k)}(\bar{z}), \tilde{s}_{y}^{(k)}(\bar{z})$. Their OPEs with the fermions are

$$
\begin{aligned}
& \psi_{x}(z) s_{x}^{(k)}(w)=(z-w)^{\frac{k}{n}} t_{x}^{(k)}(w), \\
& \bar{\psi}_{x}(z) s_{x}^{(k)}(w)=\frac{1}{(z-w)^{\frac{k}{n}}} t_{x}^{(k)}(w)
\end{aligned}
$$

and similar for the world-sheet complex conjugates. Analogous formulæ will hold for the fermions associated with the $Y$ coordinate. World-sheet complex conjugation means $(z \leftrightarrow \bar{z}, h \leftrightarrow \bar{h})$. One has $\tilde{\sigma}_{x}^{(k)}=\sigma_{x}^{(k)}$.

The spin fields can be represented by means of a bosonization:

$$
\begin{aligned}
& \psi_{x}=-i \sqrt{2} \mathrm{e}^{i H_{x}}, \quad s^{(k)}=\mathrm{e}^{i \frac{k}{n} H_{x}}, \quad \bar{\psi}_{x}=-i \sqrt{2} \mathrm{e}^{-i H_{x}}, \\
& t_{x}^{\prime(k)}=-i \sqrt{2} \mathrm{e}^{i\left(1+\frac{k}{n}\right) H_{x}}, \quad s_{x}^{(k)}=\mathrm{e}^{i \frac{k}{n} H_{x}}, \quad t_{x}^{(k)}=-i \sqrt{2} \mathrm{e}^{-i\left(1-\frac{k}{n}\right) H_{x}} .
\end{aligned}
$$

The twist operators for the bosonic fields were introduced in 29 and they are denoted by $\sigma_{x}^{(k)}(z, \bar{z})$ and $\sigma_{y}^{(k)}(z, \bar{z}), k=1, \ldots n$. In a neighborhood of a twist field located at the origin the fields $X$ and $Y$ have the monodromy properties

$$
X\left(\mathrm{e}^{2 \pi i} z, \mathrm{e}^{-2 \pi i} \bar{z}\right)=\mathrm{e}^{2 \pi i \frac{k}{n}} X(z, \bar{z}), \quad Y\left(\mathrm{e}^{2 \pi i} z, \mathrm{e}^{-2 \pi i} \bar{z}\right)=\mathrm{e}^{2 \pi i \frac{n-k}{n}} Y(z, \bar{z})
$$

Correspondingly, the OPEs of the twist fields with $\partial X(z), \partial \bar{X}(z), \bar{\partial} X(\bar{z})$ and $\bar{\partial} \bar{X}(\bar{z})$ are

$$
\begin{aligned}
\partial X(z) \sigma_{x}^{(k)}(w, \bar{w}) & =\frac{1}{(z-w)^{1-\frac{k}{n}}} \tau_{x}^{(k)}(w, \bar{w}), \\
\partial \bar{X}(z) \sigma_{x}^{(k)}(w, \bar{w}) & =\frac{1}{(z-w)^{\frac{k}{n}}} \tau_{x}^{\prime(k)}(w, \bar{w}), \\
\bar{\partial} X(\bar{z}) \sigma_{x}^{(k)}(w, \bar{w}) & =\frac{1}{(\bar{z}-\bar{w})^{\frac{k}{n}}} \tilde{\tau}_{x}^{\prime(k)}(w, \bar{w}), \\
\bar{\partial} \bar{X}(\bar{z}) \sigma_{x}^{(k)}(w, \bar{w}) & =\frac{1}{(\bar{z}-\bar{w})^{1-\frac{k}{n}}} \tilde{\tau}_{x}^{(k)}(w, \bar{w}) .
\end{aligned}
$$


The $\tau$-fields are called excited twist fields. Similar formulæ hold for the $Y$ coordinate and the corresponding twist fields. Unfortunately, we don't have an explicit construction of the bosonic twists in terms of the fundamental bosonic fields, a fact which makes more difficult the computation of correlation functions and fusion rules involving twist fields [29].

The operator content of the orbifold conformal field theory is given by $\Gamma$ invariant operators (coming from the untwisted sector) and product of twist fields and $\Gamma$ invariant operators (from the twisted sectors). From the operatorial point of view, the projection onto invariant states is needed to obtain a set of mutually local operators.

The computation of the expectation value of the stress-energy tensor in the presence of twist fields [29] gives the conformal dimension of the twist $h_{\sigma^{(k)}}=\frac{1}{2}(k / n)(1-k / n)$. From the bosonization rules (105) we learn more directly the conformal dimension of the spin field $h_{s^{(k)}}=\frac{1}{2}(k / n)^{2}$.

From the bosonization rules, we have

$$
\begin{aligned}
\psi_{x}(z) t_{x}^{(k)}(w) & =-\frac{2}{(z-w)^{1-\frac{k}{n}}} s_{x}^{(k)}(w), \\
\bar{\psi}_{x}(z) t_{x}^{\prime(k)}(w) & =-\frac{2}{(z-w)^{1+\frac{k}{n}}} s_{x}^{(k)}(w) .
\end{aligned}
$$

The other OPEs of this kind (i.e. for $\psi_{x}(z) t_{x}^{\prime(k)}(w)$ and $\left.\bar{\psi}_{x}(z) t_{x}^{(k)}(w)\right)$ are regular. The analogous formulæ for the OPEs between bosons and excited twist fields will be derived later on, when studying systematically the product of representations of the orbifold conformal field theory. Moreover, we have, for $k+k^{\prime}<n$

$$
\begin{aligned}
s_{x}^{(k)}(z) s_{x}^{\left(k^{\prime}\right)}(w) & =(z-w)^{\frac{k}{n} \frac{k^{\prime}}{n}} s_{x}^{\left(k+k^{\prime}\right)}(w), \\
s_{x}^{(n-k)}(z) s_{x}^{\left(n-k^{\prime}\right)}(w) & =\frac{i}{\sqrt{2}}(z-w)^{\frac{k}{n} \frac{k^{\prime}}{n}} \psi_{x}(z) s_{x}^{\left(n-k-k^{\prime}\right)}(w) .
\end{aligned}
$$

These formulæ will be useful in the following.

As for the OPEs between twist fields, it is reasonable to assume

$$
\begin{aligned}
\sigma_{x}^{(k)}(z, \bar{z}) \sigma_{x}^{\left(k^{\prime}\right)}(w, \bar{w}) & =\frac{C_{k, k^{\prime}}^{k+k^{\prime}}}{|z-w|^{2 \frac{k}{n} \frac{k^{\prime}}{n}}} \sigma_{x}^{\left(k+k^{\prime}\right)}(w, \bar{w}) \text { for } k+k^{\prime}<n, \\
\sigma_{x}^{(k)}(z, \bar{z}) \sigma_{x}^{\left(k^{\prime}\right)}(w, \bar{w}) & =\frac{C_{k, k^{\prime}}^{k+k^{\prime}-n}}{|z-w|^{2\left(1-\frac{k}{n}\right)\left(1-\frac{k^{\prime}}{n}\right)}} \sigma_{x}^{\left(k+k^{\prime}-n\right)}(w, \bar{w}) \text { for } k+k^{\prime}>n,
\end{aligned}
$$

where $C_{k, k^{\prime}}^{k+k^{\prime}}$ and $C_{k, k^{\prime}}^{k+k^{\prime}-n}$ are certain coefficients (a sort of structure constants) that we do not need to specify here.

We now study the representations of the orbifold theory. The representations that are defined by means of twist and spin fields and not only with the fields of the $\mathbf{C}^{2}$-theory 
will be called twisted representations. The twisted short representations mix the left and right sectors. The lowest component of the short representations are

$$
\Psi_{k}\left[\begin{array}{l}
\frac{1}{2}, \frac{1}{2} \\
\frac{1}{2}, \frac{1}{2}
\end{array}\right]^{a \tilde{b}}(z, \bar{z})=\sigma_{x}^{(k)}(z, \bar{z}) \sigma_{y}^{(n-k)}(z, \bar{z})\left[\begin{array}{l}
s_{x}^{(k)} s_{y}^{(n-k)} \\
\frac{i}{2} t_{x}^{(k)} t_{y}^{(n-k)}
\end{array}\right]^{a}(z)\left[\begin{array}{l}
\tilde{s}_{x}^{(k)} \tilde{s}_{y}^{(n-k)} \\
\frac{i}{2} \tilde{t}_{x}^{(k)} \tilde{t}_{y}^{(n-k)}
\end{array}\right]^{\tilde{b}}(\bar{z})
$$

The field content of the representation will be denoted by

$$
\left(\Psi_{k}^{a \tilde{a}},(\Psi \tilde{\Phi})_{k}^{a},(\Psi \tilde{\Pi})_{k}^{a},(\Phi \tilde{\Psi})_{k}^{\tilde{a}},(\Pi \tilde{\Psi})_{k}^{\tilde{a}},(\Phi \tilde{\Phi})_{k},(\Phi \tilde{\Pi})_{k},(\Pi \tilde{\Phi})_{k},(\Pi \tilde{\Pi})_{k}\right)
$$

The notation of the fields is reminiscent of the fact that they transform as the tensor product of two short representations [see eq.(92)], one in the left sector and one in the right sector. In this spirit we could have written $(\Psi \tilde{\Psi})_{k}^{a \tilde{a}}$ instead of $\Psi_{k}^{a \tilde{a}}$. However, we note that the twisted representations are not such a tensor product, due to the fact that the twist fields depend both on $z$ and $\bar{z}$. The operators that are needed for the description of the deformations of the conformal field theory are $(\Phi \tilde{\Phi})_{k},(\Phi \tilde{\Pi})_{k},(\Pi \tilde{\Phi})_{k}$ and $(\Pi \tilde{\Pi})_{k}$. We have to work out the required OPEs in order to get their expressions. To do this we write

$$
\begin{aligned}
\partial X(z) \tilde{\tau}_{x}^{\prime(k)}(w, \bar{w}) & =\frac{1}{(z-w)^{1-\frac{k}{n}}} \Delta \tilde{\tau}_{x}^{\prime(k)}(w, \bar{w}), \\
\partial \bar{X}(z) \tilde{\tau}_{x}^{(k)}(w, \bar{w}) & =\frac{1}{(z-w)^{\frac{k}{n}}} \bar{\Delta} \tilde{\tau}_{x}^{(k)}(w, \bar{w}), \\
\bar{\partial} X(\bar{z}) \tau_{x}^{\prime(k)}(w, \bar{w}) & =\frac{1}{(\bar{z}-\bar{w})^{\frac{k}{n}}} \tilde{\bar{\Delta}} \tau_{x}^{\prime(k)}(w, \bar{w}), \\
\bar{\partial} \bar{X}(\bar{z}) \tau_{x}^{(k)}(w, \bar{w}) & =\frac{1}{(\bar{z}-\bar{w})^{1-\frac{k}{n}}} \tilde{\Delta} \tau_{x}^{(k)}(w, \bar{w}) .
\end{aligned}
$$

The $\Delta \tau$-fields are doubly-excited twist fields. We give the explicit expressions of the fields that describe the deformations of the conformal field theory, i.e. $(\Phi \tilde{\Phi})_{k},(\Phi \tilde{\Pi})_{k}$, $(\Pi \tilde{\Phi})_{k}$ and $(\Pi \tilde{\Pi})_{k}$. Omitting the superscripts $k$ and $n-k$ in the $X$-fields and $Y$-fields, respectively, they are

$$
\begin{aligned}
(\Phi \tilde{\Phi})_{k} & =\frac{1}{2}\left[\tilde{\Delta} \tau_{x} \sigma_{y} t_{x} s_{y} \tilde{t}_{x} \tilde{s}_{y}-\tilde{\tau}_{x} \tau_{y} s_{x} t_{y} \tilde{t}_{x} \tilde{s}_{y}-\tau_{x} \tilde{\tau}_{y} t_{x} s_{y} \tilde{s}_{x} \tilde{t}_{y}+\sigma_{x} \tilde{\Delta}_{y} s_{x} t_{y} \tilde{s}_{x} \tilde{t}_{y}\right], \\
(\Phi \tilde{\Pi})_{k} & =\frac{i}{2}\left[\Delta \tilde{\tau}_{x}^{\prime} \sigma_{y} t_{x} s_{y} \tilde{s}_{x} \tilde{t}_{y}+\tau_{x} \tilde{\tau}_{y}^{\prime} t_{x} s_{y} \tilde{t}_{x} \tilde{s}_{y}-\tilde{\tau}_{x}^{\prime} \tau_{y} s_{x} t_{y} \tilde{s}_{x} \tilde{t}_{y}-\sigma_{x} \Delta \tilde{\tau}_{y}^{\prime} s_{x} t_{y} \tilde{t}_{x} \tilde{s}_{y}\right] \\
(\Pi \tilde{\Phi})_{k} & =\frac{i}{2}\left[\bar{\Delta} \tilde{\tau}_{x} \sigma_{y} s_{x} t_{y} \tilde{t}_{x} \tilde{s}_{y}-\tau^{\prime}{ }_{x} \tilde{\tau}_{y} s_{x} t_{y} \tilde{s}_{x} \tilde{t}_{y}+\tilde{\tau}_{x} \tau^{\prime}{ }_{y} t_{x} s_{y} \tilde{t}_{x} \tilde{s}_{y}-\sigma_{x} \bar{\Delta} \tilde{\tau}_{y} t_{x} s_{y} \tilde{s}_{x} \tilde{t}_{y}\right], \\
(\Pi \tilde{\Pi})_{k} & =-\frac{1}{2}\left[\tilde{\bar{\Delta}} \tau^{\prime}{ }_{x} \sigma_{y} s_{x} t_{y} \tilde{s}_{x} \tilde{t}_{y}+\tilde{\tau}_{x}^{\prime} \tau^{\prime}{ }_{y} t_{x} s_{y} \tilde{s}_{x} \tilde{t}_{y}+\tau^{\prime}{ }_{x} \tilde{\tau}_{y}^{\prime} s_{x} t_{y} \tilde{t}_{x} \tilde{s}_{y}+\sigma_{x} \tilde{\bar{\Delta}} \tau_{y}^{\prime} t_{x} s_{y} \tilde{t}_{x} \tilde{s}_{y}\right] .
\end{aligned}
$$


Consistency, i.e. the fact that the same fields of the short representation (112) can be reached from different paths when one repeatedly applies the supercurrents, implies

$$
\begin{aligned}
\partial X(z) \tilde{\tau}_{x}^{(k)}(w, \bar{w}) & =\frac{1}{(z-w)^{1-\frac{k}{n}}} \tilde{\Delta} \tau_{x}^{(k)}(w, \bar{w}), \\
\partial \bar{X}(z) \tilde{\tau}_{x}^{\prime(k)}(w, \bar{w}) & =\frac{1}{(z-w)^{\frac{k}{n}}} \tilde{\bar{\Delta}} \tau_{x}^{\prime(k)}(w, \bar{w}), \\
\bar{\partial} X(\bar{z}) \tau_{x}^{(k)}(w, \bar{w}) & =\frac{1}{(\bar{z}-\bar{w})^{\frac{k}{n}}} \Delta \tilde{\tau}_{x}^{\prime(k)}(w, \bar{w}), \\
\bar{\partial} \bar{X}(\bar{z}) \tau_{x}^{\prime(k)}(w, \bar{w}) & =\frac{1}{(\bar{z}-\bar{w})^{1-\frac{k}{n}}} \bar{\Delta} \tilde{\tau}_{x}^{(k)}(w, \bar{w}) .
\end{aligned}
$$

Eventual variants of the above representation (112), obtained by substituting in $\Psi_{k}^{1 \tilde{1}}$ the product of twist fields $s_{x}^{(k)} s_{y}^{(n-k)} \tilde{s}_{x}^{(k)} \tilde{s}_{y}^{(n-k)}$ with the product $s_{x}^{(n-k)} s_{y}^{(k)} \tilde{s}_{x}^{(k)} \tilde{s}_{y}^{(n-k)}$ or $s_{x}^{(n-k)} s_{y}^{(k)} \tilde{s}_{x}^{(n-k)} \tilde{s}_{y}^{(k)}$ or $s_{x}^{(k)} s_{y}^{(n-k)} \tilde{s}_{x}^{(n-k)} \tilde{s}_{y}^{(k)}$ and making the analogue for the $t$-fields, surely satisfy the correct OPEs. However they are not good representations of the orbifold theory, for sectors with $k \neq 0$. In fact, by definition of twisted sectors, the $X$-spin fields and $X$-twist fields must carry the same superscript, say $k$; in this case the $Y$ spin fields and $Y$-twist fields must carry the superscript $n-k$. In conclusion, the short representations of the orbifold conformal field theory are four (those of the untwisted sector) plus one for each twisted sector.

In this way we recover at the level of conformal field theory the correct counting of moduli parameters. Comparing with the abstract Hodge diamond [see eq. (四)] we see that $h^{(1,0)}=0$ and $h^{(1,1)}=4+\tau(|\tau|=n-1)$. Indeed $h^{(1,0)}=0$ is explained by the fact that the untwisted short representations $\left.\Psi_{\left[\frac{1}{2}, 0\right.}^{\frac{1}{2}, 0}\right]^{a}=\frac{1}{\sqrt{2}}\left[\begin{array}{c}\bar{\psi}_{x} \\ i \psi_{y}\end{array}\right]$ or $\Psi^{\prime}\left[\begin{array}{c}\frac{1}{2}, 0 \\ \frac{1}{2}, 0\end{array}\right]^{a}=\frac{1}{\sqrt{2}}\left[\begin{array}{c}\bar{\psi}_{y} \\ -i \psi_{x}\end{array}\right]$ of eq. (99) that are present in the $\mathbf{C}^{2}$ case, are deleted by the projection onto $\Gamma$-invariant states in the $\mathbf{C}^{2} / \Gamma$ theory. On the other hand, $h^{(1,1)}=4+\tau$ is explained by the fact that the orbifold theory contains the $|\tau|$ twisted short representations in addition to the untwisted ones. The untwisted representations correspond to the $1+3$ non-normalizable $(1,1)$-forms that have to be deleted in compact support cohomology. The abstract Hodge diamond is thus

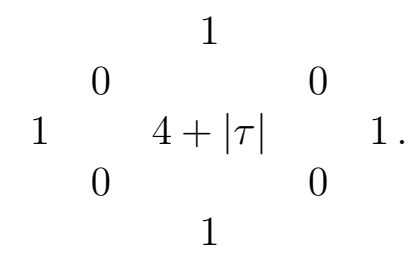

Finally, the $(4,4)$-theory corresponding to the smooth manifolds $\mathcal{M}_{\zeta}$ is obtained by perturbing the $\mathbf{C}^{2} / \Gamma$ theory with the operator

$$
\mathcal{O}=\exp \left\{\sum_{k=1}^{\tau} \int d^{2} z\left[\xi_{k}^{1}(\Phi \tilde{\Phi})_{k}+\xi_{k}^{2}(\Phi \tilde{\Pi})_{k}+\xi_{k}^{3}(\Pi \tilde{\Phi})_{k}+\xi_{k}^{4}(\Pi \tilde{\Pi})_{k}\right]\right\}
$$


where the $4 \times|\tau|$ parameters $\xi_{k}^{i}(i=1,2,3,4)$, that can be arranged into a quaternion for each value of $k$, describe the parameters of the Kähler class, complex structure and torsion deformations. In the geometric treatment we have so far considered only the HyperKähler deformations $(3 \times|\tau|$ parameters), however the conformal field theory contains also the deformations of the axion tensor $B_{\mu \nu}$ leading to the torsion deformations. The problem of identifying the moduli $t_{n}$ of (28) in terms of the moduli $\xi$ of (117) remains open. So far, we have introduced various coordinate systems in the moduli-space: the $\xi$ coordinates appearing in (117), that are a sort of "flat coordinates" and that describe all possible deformations, the $\zeta$-coordinates of the momentum map approach (see section 4) that parametrize the deformations of the complex structure and Kähler class and the $t$-coordinates that parametrize the chiral ring $\mathcal{R}=\mathbf{C}^{2}[x, y, z] / \partial W$. Formula (77) established the relation between the $t$ and the $\zeta_{+}$parameters [called $\lambda$ in the context of formula (77)]. The extension of this identification to the $\xi$ parameters is an open problem.

We conclude this section by studying the operator product of two short representations, that is the set of all the OPEs between the fields of the two multiplets. This operation is interesting, because it provides examples of twisted long representations. For simplicity, we concentrate only on the left part of the representations, thus omitting all tilded fields. Let us consider the product of an untwisted representation [say the one of (99)] with the representation (112). The untilded part of $\Psi_{k}^{a \tilde{b}}$ will be denoted by $\Psi_{k}^{a}$. The two singlets of the untilded part of representation (112) will be denoted by $\Phi_{k}$ and $\Pi_{k}$, so that the notation for the entire representation will be $\left(\Psi_{k}^{a}, \Phi_{k}, \Pi_{k}\right)$. The product in question gives a long representation in which the lowest weight primary field $\Omega_{k}$ is

$$
\Omega_{k}=\frac{1}{\sqrt{2}} \sigma_{x}^{(k)} \sigma_{y}^{(n-k)} t_{x}^{(k)} s_{y}^{(n-k)}
$$

and its weight is $h=1-k / n$. In particular, we have

$$
\Psi^{a}(z) \Psi_{k}^{b}(w, \bar{w})=\delta^{a b} \frac{1}{(z-w)^{\frac{k}{n}}} \Omega_{k}(w, \bar{w}) .
$$

One can check that consistency with the general OPEs of (96) fixes the OPEs between $\partial X, \partial \bar{X}$ and the excited twist fields. We only give some examples

$$
\begin{aligned}
\partial X(z) \tau_{x}^{(k)}(w, \bar{w}) & =\frac{\Delta \tau_{x}^{(k)}(w, \bar{w})}{(z-w)^{1-\frac{k}{n}}} \\
\partial \bar{X}(z) \tau_{x}^{(k)}(w, \bar{w}) & =-2 \frac{k}{n} \frac{1}{(z-w)^{1+\frac{k}{n}}} \sigma_{x}^{(k)}(w, \bar{w})+\frac{\bar{\Delta} \tau_{x}^{(k)}(w, \bar{w})}{(z-w)^{\frac{k}{n}}} \\
\partial X(z) \tau_{x}^{\prime(k)}(w, \bar{w}) & =-2\left(1-\frac{k}{n}\right) \frac{1}{(z-w)^{2-\frac{k}{n}}} \sigma_{x}^{(k)}(w, \bar{w})+\frac{\Delta \tau_{x}^{\prime(k)}(w, \bar{w})}{(z-w)^{1-\frac{k}{n}}} \\
\partial \bar{X}(z){\tau_{x}^{\prime(k)}}_{x}(w, \bar{w}) & =\frac{\bar{\Delta}{\tau_{x}^{\prime}}_{x}^{(k)}(w, \bar{w})}{(z-w)^{\frac{k}{n}}}
\end{aligned}
$$


for certain $\Delta \tau_{x}^{(k)}(w, \bar{w}), \bar{\Delta} \tau_{x}^{(k)}(w, \bar{w}), \Delta{\tau^{\prime}}_{x}^{(k)}(w, \bar{w})$ and $\overline{\Delta \tau^{\prime}}{ }_{x}^{(k)}(w, \bar{w})$. Going on in this way, OPEs involving doubly excited twist fields can also be found.

We now consider the operator product of two short representations of the twisted sectors. Using (109) and (110), we find that this product gives one of the twisted long representations that we have just found. So, the product of two twisted short representations of the orbifold is also equal to the product of an untwisted short representation and a third twisted short representation of the orbifold. Precisely,

$$
R_{k}(z, \bar{z}) R_{k^{\prime}}(w, \bar{w})=R_{0}^{\epsilon_{k+k^{\prime}}}(z, \bar{z}) R_{k+k^{\prime} \bmod n}(w, \bar{w}),
$$

where $R_{k}(z, \bar{z})$ denotes in compact form the twisted massless representation of the $k^{\text {th }}$ sector, while $R_{0}^{\epsilon_{k+k^{\prime}}}(z, \bar{z})$ is one of the two untwisted short representations, which of them depending on the sign of $k+k^{\prime}-n$.

In this way we can define a natural product between the short representations of the orbifold, that still gives a short representation of the orbifold and that satisfies the cyclic property of the $A_{n-1}$ subgroup of $S U(2)$, namely

$$
R_{k} \otimes R_{k^{\prime}}=R_{k+k^{\prime} \bmod n}
$$

The last remark we make concerns the chiral ring [6] of the conformal field theory under consideration. Let

$$
\mathcal{O}_{k}=\sigma_{x}^{(k)} \sigma_{y}^{(n-k)} s_{x}^{(k)} s_{y}^{(n-k)} \tilde{s}_{x}^{(k)} \tilde{s}_{y}^{(n-k)}
$$

be the operator that, acting on the vacuum, gives the vacuum of the $k^{\text {th }}$ twisted sector. Viewing the $\mathrm{N}=4$ theory as an $\mathrm{N}=2$ theory, the $\mathcal{O}_{k}$ are chiral operators. All of them have charge 1 (this is the $U(1)$ charge corresponding to the current $A_{3}(z)$ ). There must also exist a unique chiral operator of charge $\frac{c}{3}=2$ and conformal weight 1 . This operator is $A^{+}$. These operators $\left(A^{+}, \mathcal{O}_{k}\right)$ together with the identity span the chiral ring $\mathcal{C}$ of the $N=2$ theory, which happens to have only integral $U(1)$ charges. As a matter of fact, one verifies that

$$
\begin{aligned}
\mathcal{O}_{k} \mathcal{O}_{k^{\prime}} & =0 & \text { if } k+k^{\prime} \neq n \\
\mathcal{O}_{k} \mathcal{O}_{n-k} & \sim A^{+} . &
\end{aligned}
$$

It is important to stress that this $\mathrm{N}=2$ chiral ring is not the $\operatorname{ring} \mathcal{R}=C[x, y, z] / \partial W$, although the short representations are in one-to-one correspondence with the elements of $\mathcal{R}$. It remains a so far unsolved problem to interpret the multiplication rule of $\mathcal{R}$ within the conformal field theory. In particular, we can perform the standard topological twist; the chiral ring $\mathcal{C}$ coincides then with the set of physical fields of the topological model. The multiplication table (124) of the unperturbed chiral ring $\mathcal{C}$ yields the two point function (the topological metric) which is just constant in parameter space. One can also perturb the topological model by inserting into the correlators the exponential of the integrated physical fields, namely the second components of the $\mathcal{O}_{k}$ chiral multiplets. 
This is nothing else but the operator $\mathcal{O}$ in eq.(117). As it is well known the parameters $\xi$ of the perturbation of a topological model around its conformal point are the flat coordinates (see [31] whose relation with the parameters $t$ in the deformed Landau-ginzburg potential (if one exists) is given by the solution of a complicated but well-defined uniformization problem. Therefore if the chiral ring $\mathcal{C}$ of the $N=2$ were identified with the chiral ring $\mathcal{R}$ then the relation between the $x_{i}$ parameters in eq.(117) and the $t$-parameters in eq.s (28,77) could be retrieved using the methods developed for Topological LandauGinzburg models. This shows that finding the relation between $\xi$ and $t$ or finding the relation between the two rings $\mathcal{C}$ and $\mathcal{R}$ or finding the relation between the $\sigma$-model on an ALE space and a suitable Landau-Ginzburg model are just three different aspects of the same problem.

\section{$6 \quad$ Partition function and $N=4$ characters}

As stressed several times the moduli of a $(4,4)$ theory are the highest components of short representations. The characters of the short representations were computed by Eguchi and Taormina in [32]. They read [5:

$$
\begin{aligned}
& \operatorname{ch}_{0}^{N S}(l=0 ; \tau ; z)=2\left(\frac{\theta_{1}(z)}{\theta_{3}(0)}\right)^{2}+\left(\frac{q^{-\frac{1}{8}}}{\eta(\tau)}-2 h_{3}(\tau)\right)\left(\frac{\theta_{3}(z)}{\eta(\tau)}\right)^{2} \\
& \operatorname{ch}_{0}^{N S}\left(l=\frac{1}{2} ; \tau ; z\right)=-\left(\frac{\theta_{1}(z)}{\theta_{3}(0)}\right)^{2}+h_{3}(\tau)\left(\frac{\theta_{3}(z)}{\eta(\tau)}\right)^{2}
\end{aligned}
$$

where $h_{3}$ is defined by

$$
h_{3}(\tau)=\frac{1}{\eta(\tau) \theta_{3}(0)} \sum_{m} \frac{q^{\frac{1}{2} m^{2}-\frac{1}{8}}}{1+q^{m-\frac{1}{2}}}
$$

The characters of the long representations are instead given by:

$$
c h^{N S}(h ; \tau ; z)=\frac{q^{h-\frac{1}{8}}}{\eta(\tau)}\left(\frac{\theta_{3}(z)}{\eta(\tau)}\right)^{2}
$$

The above formulae apply to a general $\mathrm{N}=4$ theory. We are interested in the specific case of the orbifold theories discussed in the previous section. We want to explore their spectrum by investigating their partition function and by decomposing it into characters of the $\mathrm{N}=4$ algebra.

Consider the partition function for the $A_{k-1}$ models; we have to sum contributions from the twisted sector, i.e. to sum over different boundary conditions in the spatial direction. To obtain a modular invariant partition function, we have to twist also in the time direction. As we have already explained in section 5, the twisted boundary conditions

\footnotetext{
${ }^{3}$ in the following we use for the theta-functions with characteristic two equivalent notations: $\theta\left[\begin{array}{c}0 \\ 0\end{array}\right] \equiv \theta_{3}$, $\theta\left[\begin{array}{l}1 \\ 0\end{array}\right] \equiv \theta_{2}, \theta\left[\begin{array}{l}0 \\ 1\end{array}\right] \equiv \theta_{4}, \theta\left[\begin{array}{l}1 \\ 1\end{array}\right] \equiv \theta_{1}$.
} 
are associated with the non-trivial conjugacy class of the group. In the abelian case $A_{k-1}$, we have a conjugacy class for every element of the cyclic group; therefore the boundary conditions are parametrized by an integer $0 \leq i \leq k-1$. If we denote $\nu=e^{2 \pi i / k}$, the building blocks for the partition function are

$$
Z_{r, s}=(q \bar{q})^{-c / 24} \operatorname{Tr}_{\nu^{r}} \hat{\nu}^{s} q^{L-0} \bar{q}^{\bar{L}_{0}} e^{2 \pi i z J_{3}+2 \pi i \bar{z} J_{3}}
$$

where $\hat{\nu}$ is the operator on the Hilbert space which implements the action of the generator of $A_{k-1} . Z_{r, s}$ is the partition function twisted by $\nu^{r}$ in the spatial direction and by $\nu^{s}$ in the temporal one.

A modular invariant partition function can be constructed by summing over all boundary conditions

$$
Z=\frac{1}{k} \sum_{r, s} Z_{r, s} C_{r, s}
$$

with coefficients $C_{r, s}=1$. In terms of Hilbert space states, the sum over the spatial conditions takes into account the existence of several sectors of the Hilbert space, while the sum over temporal conditions, at fixed spatial ones, realizes the necessary projection of the theory onto group invariant states.

We have to sum over the twisted boundary conditions for bosons and fermions dictated by the orbifold construction and, independently, over the four spin structures of fermions to take into account the existence of Ramond (R) and Neveu-Schwarz (NS) sectors. To compute the total partition function [33] we begin with the contribution from the untwisted sector. Decomposing the fields $X, Y, \psi_{x}, \psi_{y}$ in Fourier modes $(\alpha, \beta, \lambda, \mu)$ with the commutation relations

$$
\begin{aligned}
& {\left[\bar{\alpha}_{n}, \alpha_{m}\right]=n \delta_{n+m, 0}} \\
& \left\{\bar{\lambda}_{n}, \lambda_{m}\right\}=\delta_{n+m, 0}
\end{aligned}
$$

and similar for $\beta, \mu$, we implement the group transformation on the Hilbert space via

$$
\left(\alpha_{n}, \bar{\beta}_{n}, \lambda_{n}, \bar{\mu}_{n}\right) \rightarrow e^{2 \pi i / k}\left(\alpha_{n}, \bar{\beta}_{n}, \lambda_{n}, \bar{\mu}_{n}\right)
$$

In the untwisted sector we take a basis of eigenvectors of $L_{0}, \alpha_{0}, \beta_{0}$ to perform the trace on Hilbert space; note that in this sector the zero modes of bosonic fields (the momentum) commute with $L_{0}$. The Fock space is constructed by applying the raising operators to the vacuum and to the eigenvectors of the momentum $\left|p_{x}, p_{y}\right\rangle$. The trace with $\hat{\nu}$ inserted picks out contributions only from the vacuum, because $\hat{\nu}$ is not diagonal on the momentum eigenvectors. The computation is now straightforward [33] and reduces to the computation of the partition function of free bosons and fermions with twisted boundary conditions. The contribution of the unprojected trace is the partition function of the flat $\mathrm{N}=4$ space

$$
\frac{1}{k} \frac{1}{(\eta \bar{\eta})^{4}} \int d p_{x} d p_{y} q^{p_{x} \bar{p}_{x}+p_{y} \bar{p}_{y}} \frac{1}{2} \sum\left|\frac{\theta\left[\begin{array}{l}
a \\
b
\end{array}\right](z)}{\eta}\right|^{4} .
$$


In the sector r-times twisted in the temporal direction, the standard $\eta$ function of a boson (for example) is replaced by the following infinite product

$$
q^{-\frac{1}{24}} \frac{1}{\prod_{n=1}^{\infty}\left(1-\nu^{r} q^{n}\right)}
$$

In the twisted sector of the theory the Hilbert space is constructed by the application of oscillators to the twisted vacua, obtained by applying the twist fields to the true vacuum. This explains a further factor of $q^{1 / 2}$ to the energy due to the conformal dimension of the twist fields $O_{i}$ (see eq. 123). The oscillators have now fractional indices and so they contribute fractional powers of $q$ in the infinite product which replaces the $\eta$ function, exactly as it happens to free fermions when we change the spin structure (i.e. boundary conditions). From

$$
i \partial X=\sum_{n} \frac{\alpha_{n}}{z^{n+1}}, \quad i \psi_{x}=\sum_{n} \frac{\lambda_{n}}{z^{n+1 / 2}}(N S), \ldots
$$

and the transformation $\partial X \rightarrow e^{2 \pi i s / k} \partial X, \ldots$ under the group $A_{k-1}$ we learn the modings

$$
\begin{array}{ll}
\alpha_{n}, \bar{\beta}_{n}, \lambda_{n}, \bar{\mu}_{n}(P), & n \epsilon Z-\frac{s}{k} \\
\bar{\alpha}_{n}, \beta_{n}, \bar{\lambda}_{n}, \mu_{n}(P), & n \epsilon Z+\frac{s}{k} .
\end{array}
$$

Collecting all these informations the general contribution from antiperiodic-antiperiodic fermions (to give an explicit example) is

$$
\begin{aligned}
q^{-2 / 24} & \prod_{n=0}^{\infty}\left(1+e^{2 \pi i z} \nu^{r} q^{n+\frac{s}{k}+\frac{1}{2}}\right)\left(1+e^{2 \pi i z} \bar{\nu}^{r} q^{n+\frac{k-s}{k}-\frac{1}{2}}\right) \times \\
& \times\left(1+e^{-2 \pi i z} \nu^{r} q^{n+\frac{s}{k}+\frac{1}{2}}\right)\left(1+e^{-2 \pi i z} \bar{\nu}^{r} q^{n+\frac{k-s}{k}-\frac{1}{2}}\right)
\end{aligned}
$$

from the triple product identity [33

$$
\prod_{n=0}^{\infty}\left(1-q^{n+1}\right)\left(1+q^{n+1 / 2} \omega\right)\left(1+q^{n+1 / 2} \omega^{-1}\right)=\sum_{n \in Z} q^{n^{2} / 2} \omega^{n}
$$

with $\omega=\nu^{r} q^{s / k}$ we obtain

$$
\begin{aligned}
& q^{1 / 24}\left(1+e^{2 \pi i z} \nu^{r} q^{n+\frac{s}{k}+\frac{1}{2}}\right)\left(1+e^{2 \pi i z} \bar{\nu}^{r} q^{n+\frac{k-s}{k}-\frac{1}{2}}\right)= \\
& =\frac{1}{\eta(q)} \sum_{Z} q^{n^{2} / 2} \nu^{n r} e^{2 \pi i n z} q^{n s / k}=\frac{\theta_{3}(z+(r+s \tau) / k)}{\eta} .
\end{aligned}
$$

The introduction of the fermionic spin structures simply shifts some parameters in the $\theta$ function. For simplicity, we collect only the results for the NS sector. Fermions contribute two factors like the one above, while for the same reasoning bosons (collecting with care 
also the $q^{1 / 2}$ factor in the twisted sector) contribute a factor $\frac{\eta^{2}}{\theta_{1}}$. The building blocks (in the NS sector) of the partition function are, for a fixed fermionic spin structure, say $\left[\begin{array}{l}0 \\ 0\end{array}\right]$,

$$
Z_{r, s}\left[\begin{array}{l}
0 \\
0
\end{array}\right]=\frac{\theta_{3}(z+(r+s \tau) / k) \theta_{3}(z-(r+s \tau) / k)}{\theta_{1}((r+s \tau) / k)^{2}}
$$

With the same technique we can compute the partition function for all boundary conditions $Z\left[\begin{array}{l}a \\ b\end{array}\right]$ and from these the analogous of the $i=(0, v, s, \bar{s})$ characters for the flat space and finally the heterotic partition function (see 4 ])

$$
Z=\sum_{i, \bar{\imath}} Z_{i, \bar{\imath}}^{(9,9)} Z_{i, \bar{\imath}}^{(6,6)} B_{i}^{(-2)}\left(B_{\bar{\imath}}^{\left(E_{8}^{\prime} \times S O(6)\right)}\right)^{*}
$$

The next step to obtain the spectrum of the theory is to decompose the partition function into characters of the $\mathrm{N}=4$ algebra.

If we are only interested in the field content of the orbifold conformal field theory (ignoring internal dimensions), the $Z\left[\begin{array}{l}a \\ b\end{array}\right]$ have to be summed with certain coefficients to obtain a modular invariant "partition function" :

$$
Z\left[\begin{array}{l}
a \\
b
\end{array}\right]=Z\left[\begin{array}{l}
a \\
b
\end{array}\right]_{\text {flat space }}+\sum_{r, s}\left|Z_{r, s}\left[\begin{array}{l}
a \\
b
\end{array}\right]\right|^{2}
$$

In the Eguchi-Hanson $(A-1)$ case the above partition function (at $z=0$, summed over all boundary conditions) is easily computed:

$$
Z_{E H}=\frac{1}{4} \frac{1}{(\eta \bar{\eta})^{4}} \int d p_{x} d p_{y} q^{p_{x} \bar{p}_{x}+p_{y} \bar{p}_{y}} \sum_{i}\left|\frac{\theta_{i}}{\eta}\right|^{4}+\frac{1}{4} \sum_{i, j}\left|\frac{\theta_{i}}{\theta_{j}}\right|^{4}
$$

It is modular invariant by inspection.

In the same spirit, the flat space contribution is

$$
Z_{\text {flat space }}=\frac{1}{|I m \tau|^{2}|\eta|^{8}} \sum_{i} \frac{1}{2}\left|\frac{\theta_{i}}{\eta}\right|^{4}
$$

The momentum integral annihilates the contribution of short representation which are a zero measure set with respect to the continuum spectrum. We expect that the flat space partition function is an integral over the continuum spectrum of the theory of long representations with characters

$$
c h^{N S}=\frac{q^{h-1 / 8}}{\eta} \frac{\theta_{3}^{2}}{\eta^{2}}
$$

The continuum spectrum is realized by exponential fields or exponential fields multiplied by combinations of derivatives of the bosonic fields and the singlets that we can realize 
with fermions such that the entire field is an $\mathrm{N}=4$ primary. A combinatorial computation shows that this sum reconstructs exactly the three factors of $\eta$ needed to obtain $Z_{\text {flat space }}$

The twisted sectors $Z_{s, r}$ do not receive contributions from the continuum spectrum given by the exponentials. In the Eguchi-Hanson case the explicit form of $Z_{r, s}$ at $z \neq 0$ is

$$
\begin{aligned}
& Z_{01}(z)=\left(\frac{\theta_{1}(z)}{\theta_{3}}\right)^{2}-\left(\frac{\theta_{2}}{\theta_{4}}\right)^{2}\left(\frac{\theta_{3}(z)}{\theta_{3}}\right)^{2} \\
& Z_{10}(z)=-\left(\frac{\theta_{1}(z)}{\theta_{3}}\right)^{2}-\left(\frac{\theta_{4}}{\theta_{2}}\right)^{2}\left(\frac{\theta_{3}(z)}{\theta_{3}}\right)^{2} \\
& Z_{11}(z)=-\left(\frac{\theta_{1}(z)}{\theta_{3}}\right)^{2}-\left(\frac{\theta_{1}}{\theta_{3}}\right)^{2}\left(\frac{\theta_{3}(z)}{\theta_{3}}\right)^{2}
\end{aligned}
$$

The contribution of the spatial twisted sectors is given by the sum of the character of the short representation which perform the twist and an infinite number of long representations, while in the sector twisted only in the time direction we obtain, as one can expect, the short representation corresponding to the identity plus long characters.

For example

$$
\begin{aligned}
& Z_{10}=c h_{0}^{N S}(l=1 / 2, z)-\left(h_{3}+\frac{\theta_{4}^{4}}{4 \eta^{4}}\right)\left(\frac{\theta_{3}(z)}{\eta}\right)^{2}= \\
& \operatorname{ch}_{0}^{N S}(l=1 / 2, z)+\sum_{i} A_{i} q^{i} \frac{q^{t-1 / 8}}{\eta}\left(\frac{\theta_{3}(z)}{\eta}\right)^{2} \\
& =\operatorname{ch}_{0}^{N S}(l=1 / 2, z)+\sum_{i} A_{i} c h^{N S}(h=t+i, l=0)
\end{aligned}
$$

where $A_{i}, t$ are coefficients in the expansion of $h_{3}, \eta, \theta$ in powers of q. This decomposition of the partition function agrees with the previous discussion and it explicitly shows the appearance of a number of massless representation related to the Hirzebruck signature $\tau$.

\section{Comparison with the $S U(2) \times \mathbf{R}$ instanton and con- cluding remarks}

The conformal field theory based on the supersimmetrization of the direct product of a $S U(2)$ WZNW theory at level $k$ and a Feigin-Fuchs (F.F.)boson with $k$-dependent background charge has been widely discussed in the literature [34, 9, 7, , 4, 5]. One basic feature is that the four fermions $\lambda^{0}$ (the superpartner of the F.F. boson $\partial t$ ) and $\lambda^{i}$, $i=1,2,3$ (the superpartners of the $S U(2)_{k}$ currents) constitute a system of free fermions. Another fundamental point is that if the background charge of the F.F. boson takes the 
value $-i \sqrt{\frac{2}{k+2}}$, the total central charge of the theory results to be

$$
c=c_{W Z W}+c_{F . F .}+c_{\text {freefermions }}=\frac{3 k}{k+2}+1+\frac{6}{k+2}+2=6
$$

and that the theory actually admits $N=4$ superconformal symmetry. As emphasized in [3] this makes exact (to all orders in $\alpha^{\prime}$ ) the correspondence of this CFT with the $\sigma$-model formulated on a certain four-dimensional target space, a particular limit of a gravitational instanton with torsion [3, 4.

In [4] we focused our attention on the abstract Hodge diamond [see (4)] of the theory, and we identified four $(1,1)$ abstract forms (giving rise to moduli of the theory) $\Psi_{A}\left[\begin{array}{c}\frac{1}{2}, \frac{1}{2} \\ \frac{1}{2}, \frac{1}{2}\end{array}\right]^{m, \tilde{m}}(z, \bar{z})$. However, as remarked by C.Kounnas in [5], more moduli can be written. The abstract Hodge diamond shows thus a more interesting structure; we briefly describe it. In particular it turns out that the abstract Hodge diamond (that is intimately related, as we have stressed throughout all the paper, to the topology of the corresponding four-space) is sensitive to the choice of the modular invariant for the $S U(2)_{k}$ subtheory.

The fundamental fields of the theory, that is the F.F. boson $t$, the $S U(2)_{k}$ currents $J^{i}$ and the free fermions $\lambda^{a}, a=0,1,2,3$, are normalized as follows t:

$$
\begin{aligned}
\partial t(z) \partial t(w) & =\frac{-1}{(z-w)^{2}}, \quad \lambda^{a}(z) \lambda^{b}(w)=-\frac{1}{2} \frac{\delta^{a b}}{z-w}, \\
J^{i}(z) J^{j}(w) & =\frac{k}{2} \frac{\delta^{i j}}{(z-w)^{2}}+i \frac{\epsilon^{i j k} J^{k}}{z-w} .
\end{aligned}
$$

The $S U(2)$ currents $A^{i}$ of the $N=4$ algebra eq.(85) are purely fermionic, and are therefore identical to those of the flat four-space eq. $(98)$ :

$$
A^{i}=i\left(\lambda^{0} \lambda^{i}+\frac{1}{2} \epsilon^{i j k} \lambda^{j} \lambda^{k}\right)
$$

Denote as $\Theta_{1}=\left(\lambda^{0}+i \lambda^{3}, \lambda^{2}+i \lambda^{1}\right), \Theta_{2}=\left(\lambda^{2}-i \lambda^{1},-\left(\lambda^{0}-i \lambda^{3}\right)\right)$ the two possible doublets of fermions. The supercurrents are then

$$
\begin{aligned}
& \mathcal{G}=\left(\partial t-\sqrt{\frac{2}{k+2}}\left[J^{3}-2 i\left(\lambda^{0} \lambda^{3}-\lambda^{1} \lambda^{2}\right)-\partial\right]\right) \Theta_{1}+\sqrt{\frac{2}{k+2}} J^{-} \Theta_{2}, \\
& \overline{\mathcal{G}}=\left(\partial t-\sqrt{\frac{2}{k+2}}\left[J^{3}+2 i\left(\lambda^{0} \lambda^{3}-\lambda^{1} \lambda^{2}\right)-\partial\right]\right) \Theta_{1}^{*}+\sqrt{\frac{2}{k+2}} J^{+} \Theta_{2}^{*} .
\end{aligned}
$$

The primary fields : $e^{i \alpha t}$ : of the F.F. theory have conformal weight $\Delta_{\alpha}=\frac{1}{2} \alpha(\alpha-$ $i \sqrt{2 / k+2})$. The primary fields corresponding to the integrable representations for

\footnotetext{
${ }^{4}$ We recall the expressions and normalizations of the fields only in the left sector; those in the right one are almost identical. As already remarked, however, non-trivial ways of merging together left and right sector will be significative.
} 
$S U(2)$ at level $k$, i.e. those of spin $l=0, \frac{1}{2}, 1, \ldots, \frac{k}{2}$, have dimensions $h_{l}=\frac{l(l+1)}{k+2}$; we denote them as $\phi_{m}^{l}, m$ being the third component (the eigenvalue of $J^{3}$ ). It is then possible to verify that the following doublets satisfy the basic OPEs (92) so that they give rise to short representations in the left sector:

$$
\begin{aligned}
& \Psi_{1}^{(l)}=e^{-\sqrt{2 / k+2}(l+1) t} \phi_{-l}^{l} \Theta_{1}, \\
& \Psi_{2}^{(l)}=e^{-\sqrt{2 / k+2}(l+1) t} \phi_{l}^{l} \Theta_{2} .
\end{aligned}
$$

To check the OPEs (92) one has to recall the action of the third-component $J^{3}$ and of the raising and lowering operators $J^{ \pm}$on the $S U(2)$ primary fields, which is:

$$
\begin{aligned}
J^{3}(z) \phi_{m}^{l}(w) & =m \frac{\phi_{m}^{l}}{z-w}, \\
J^{ \pm}(z) \phi_{m}^{l}(w) & =N_{m}^{l} \frac{\phi_{m \pm 1}^{l}}{z-w},
\end{aligned}
$$

where $N_{m}^{l}=\sqrt{l(l+1)-m(m \pm 1)}$. It is immediate to verify the the $\Psi_{A}^{(l)}$ fields have weight $\frac{1}{2}$, as this is the weight of the fermions and the negative weight of $e^{-\sqrt{2 / k+2}(l+1) t}$ cancels that of $\phi_{m}^{l}$. It is also straightforward to write down the highest components $\Phi_{A}^{(l)}$ and $\Pi_{A}^{(l)}$ of these short representations.

If we want to find out the numbers of abstract $(p, q)$-forms we must specify how to put together the two sectors of the theory. The basic constraint we have to satisfy is the modular invariance of the partition function. In [4] we focused on the complete partition function for the heterotic string moving on a background given by six compactified dimensions times the four ones we are discussing. What matters here of that discussion (see also section (6) is that we are interested in writing the partition function for fixed spin structures of the $(4,4), c=6$ theory, $Z_{i, \bar{\imath}}^{(6,6)}$. The index $i$ denotes the $S O(4)$ characters $0, v, s, \bar{s}$. Since the four fermions $\lambda^{a}$ are effectively free, they take care of these characters. Then the other two subtheories, namely $S U(2)_{k}$ and the F.F. boson, must correspond by themselves to modular invariant partition functions of Of course the very constrained part is the $S U(2)_{k}$ theory, for which the possible modular invariants are well-known. These invariants depend on the level $k$ and are related to the ADE classification [35]. The invariant partition functions given in Table 3 , where $\chi_{l}, l=0, \frac{1}{2}, \ldots \frac{k}{2}$ denotes the affine character corresponding to the representation of spin $l$.

In building the abstract $(1,1)$-forms of the theory we must take into account the fact that indeed the F.F. boson $t$ is a function of $z$ and $\bar{z}$; it is interpreted as a non-compact coordinate and we are therefore not allowed to have independent left and right momenta:

\footnotetext{
${ }^{5}$ The situation is somehow analogous to that of section 6 . Leaving aside the fermionic spin structures, we had there to obtain a modular invariant partition function for the orbifold theory as in eq.(129). Here, having as basic building blocks the affine characters $\chi_{l}$, a modular invariant partition function eq.(153) describes the $\mathrm{b} S U(2)_{k}$ theory.
} 
Table 3: Modular invariant partition functions for $S U(2)_{k}$

\begin{tabular}{|c|c|c|}
\hline Level & $\begin{array}{c}\text { ADE } \\
\text { (rank) }\end{array}$ & Partition function \\
\hline$k \geq 1$ & $\begin{aligned} & A_{k+1} \\
r= & k+1\end{aligned}$ & $\sum_{l=0, \frac{1}{2}, \ldots}^{\frac{k}{2}}\left|\chi_{l}\right|^{2}$ \\
\hline $\begin{array}{c}k=4 n-2 \\
n \geq 1\end{array}$ & $\begin{aligned} & D_{\frac{k}{2}+1} \\
r= & \frac{k}{2}+1\end{aligned}$ & $\sum_{l, i n t=0}^{\frac{k}{2}-1}\left|\chi_{l}+\chi_{\frac{k}{2}-l}\right|^{2}+2\left|\chi_{\frac{k}{4}}\right|^{2}$ \\
\hline $\begin{array}{c}k=4 n-2 \\
n \geq 2\end{array}$ & $\begin{aligned} & D_{\frac{k}{2}+2} \\
& r= \frac{k}{2}+2 \\
&\end{aligned}$ & $\sum_{l, \text { int } .=0}^{\frac{k}{2}}\left|\chi_{l}\right|^{2}+\left|\chi_{\frac{k}{2}+1}\right|^{2}+\sum_{l, \text { half } \text { int } .=\frac{1}{2}}^{\frac{k}{4}-1}\left(\chi_{l}^{*} \chi_{\frac{k}{2}-l}+\chi_{\frac{k}{2}-l}^{*} \chi_{l}\right)$ \\
\hline$k=10$ & $\begin{array}{c}E_{6} \\
r=6\end{array}$ & $\left|\chi_{0}+\chi_{3}\right|^{2}+\left|\chi_{\frac{3}{2}}+\chi_{\frac{7}{2}}\right|^{2}+\left|\chi_{2}+\chi_{5}\right|^{2}$ \\
\hline$k=16$ & $E_{7}$ & $\begin{array}{c}\left|\chi_{0}+\chi_{8}\right|^{2}+\left|\chi_{2}+\chi_{6}\right|^{2}+\left|\chi_{3}+\chi_{5}\right|^{2}+\left|\chi_{4}\right|^{2}+ \\
+\left[\left(\chi_{1}+\chi_{7}\right)^{*} \chi_{4}+\chi_{4}^{*}\left(\chi_{1}+\chi_{7}\right)\right]\end{array}$ \\
\hline$k=28$ & $E_{8}$ & $\left|\chi_{0}+\chi_{5}+\chi_{9}+\chi_{14}\right|^{2}+\left|\chi_{3}+\chi_{6}+\chi_{8}+\chi_{11}\right|^{2}$ \\
\hline
\end{tabular}

the possible operators are just $\exp (\alpha t(z, \bar{z}))$. Moreover, we can put together a field $\phi_{m_{1}}^{l_{1}}(z)$ with $\tilde{\phi}_{m_{2}}^{l_{2}}(\bar{z})$ only if, in the chosen modular invariant partition function for the $S U(2)_{k}$ subtheory

$$
\sum_{l, l^{\prime}} n_{l, l^{\prime}} \chi_{l}^{*} \chi_{l^{\prime}}
$$

the coefficient $n_{l_{1}, l_{2}}$ is different from zero.

These two considerations restrict the possible abstract $(1,1)$-forms to be:

$$
\Psi_{(A B)}^{(l)}\left[\begin{array}{c}
\frac{1}{2}, \frac{1}{2} \\
\frac{1}{2}, \frac{1}{2}
\end{array}\right]^{a, \tilde{b}}=e^{(l+1) \sqrt{2 / k+2} t(z, \bar{z})} \phi_{m_{A}}^{l}(z) \Theta_{A}^{a}(z) \tilde{\phi}_{m_{B}}^{l}(z) \tilde{\Theta}_{B}^{\tilde{b}}(\bar{z})
$$

for each spin $l$ such that the diagonal coefficient $n_{l, l}$ is non-zero in the chosen $S U(2)_{k}$ modular invariant. The indices $A, B$, labelling the distinct possible doublets of fermions take the values 1,2 , so that for each $l$ there are four different abstract $(1,1)$ forms. The third components $m_{A}$ are given by $m_{A}=(-1)^{A} l_{A}$, see eq.(151). Tildes are used to denote fields of the right sector.

One can also see that no abstract $(1,0)$ form $\left.\Psi_{\left[\frac{1}{2}, 0\right.}^{\left[\frac{1}{2}, 0\right.}\right]^{a}(z, \bar{z})$ can be constructed, as the 
possible candidates

$$
\Psi_{(A B)}^{(l)}\left[\begin{array}{c}
\frac{1}{2}, 0 \\
\frac{1}{2}, 0
\end{array}\right]^{a}=e^{(l+1) \sqrt{2 / k+2} t(z, \bar{z})} \phi_{m_{A}}^{l}(z) \Theta_{A}^{a}(z) \tilde{\phi}_{m_{B}}^{l}(\bar{z})
$$

do not show the correct behaviour in the right sector.

Recall that the number of diagonal terms in each of the possible modular invariants has a key meaning in their ADE classification: it equals the rank $r$ of the simply-laced Lie algebra $\mathcal{G}$ which corresponds to it as in Table 3. We can therefore summarize the above remarks by writing the abstract Hodge diamond of the $S U(2)_{k} \times \mathbf{R}$ theory, having chosen one of the possible partition functions for $S U(2)_{k}$ :

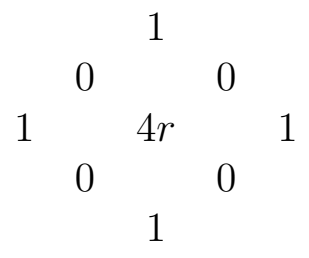

For example, if we are considering the theory $S U(2)_{10} \times \mathbf{R}$, i.e. we set $k=10$, then we can choose three different modular invariants, corresponding in the ADE classification to $A_{11}, D_{7}$ or $E_{6}$. Since the rank of these Lie algebras is respectively 11,7 and 6 , the number $h^{1,1}$ of abstract $(1,1)$ forms is very different in the three cases; respectively we have $h^{1,1}=44, h^{1,1}=28$ or $h^{1,1}=24$.

We must recall however that the four-space corresponding to the $S U(2) \times \mathbf{R}$ theory is a gravitational instanton with torsion. It admits three complex structures that are covariantly constant with respect to connections with torsion; the same remarks apply to its three HyperKähler forms (such spaces were named in [4] generalized HyperKähler spaces). In attributing a geometrical meaning to the abstract Hodge diamond (156) we must be aware that the cohomology involved is that of the fiber bundle whose connections are the torsionful connections relevant in the generalized HyperKähler case.

The analysis in this last section makes more precise some aspects of the treatment of the $S U(2) \times \mathbf{R}$ instanton that was given in [4; in particular we considerer there only 4 of the possible abstract $(1,1)$ forms, i.e the short representations $\Psi_{A B}^{(0)}\left[\begin{array}{l}\frac{1}{2}, \frac{1}{2} \\ \frac{1}{2}, \frac{1}{2}\end{array}\right]$ containing the identity in the $S U(2)_{k}$ subtheory. These are present at any level $k$, for any chosen modular invariant. Moreover we proposed to count two abstract $(1,0)$ forms, but as seen above it seems correct not to consider them; the reason is the non-compactness of the coordinate $t$ which forbids to have different momenta in left and right sector.

\section{Concluding remarks}

In conclusion, our analysis shows that one can study the family of conformal field theories associated with a parametrized family of ALE manifolds $\mathcal{M}_{\zeta}$ by deforming the exactly solvable orbifold conformal field theories $\mathbf{C}^{2} / \Gamma$. The main open problem in this respect has been pointed out at the end of section 5 . It relates with a proper interpretation 
of the chiral ring $\mathbf{C}[x, y, z] / \partial W$ within the conformal field theory. The comparison of the abstract Hodge diamond (116) of ALE theories with that of $S U(2) \times \mathbf{R}$ theories (156) shows that in general they are very different theories, having a $4 \times \tau$ and $16 \times r$ dimensional moduli space respectively. An unsolved problem is the geometrical characterization of the $16 \times r$-parameter family of generalized HyperKähler manifolds that reduces to the $S U(2) \times \mathbf{R}$ instanton of [3] at a special point.

\section{Acknowledgements}

We are very grateful to F.Gliozzi, C.Kounnas, C.Reina for clarifying discussions.

\section{References}

[1] M.Roček, K.Schoutens and A.Sevrin, Phys. Lett. B265 (1991) 303; M.Roček and E.Verlinde, Nucl. Phys. 373 (1992) 630; C.Kounnas and D.Luest, Phys. Lett. B289 (1992) 56; P.Ginsparg and F.Quevedo, Nucl. Phys. B385 (1992) 527.

[2] E. Witten, Phys. Rev. D44 (1991); R.Dijkgraf, E.Verlinde and H.Verlinde, Nucl. Phys. B371 (1992) 269.

[3] C.G.Callan, J.A.Harvey, A.Strominger, Nucl. Phys. B359 (1991) 611; C.G.Callan, Lectures at the $6^{\text {th }}$ J.A.Swieca summer school, PUTP-1728.

[4] M.Billó, P.Fré, L.Girardello and A.Zaffaroni, SISSA 159/92/EP, IFUM/431/92/FT, hep-th/9210076 (to appear in Int. Jour. Mod. Phys).

[5] C.Kounnas, "Construction of string solutions around non-trivial backgrounds" CERN-TH 6790/93; Talk given at the $26^{\text {th }}$ Workshop "From Superstrings to Supergravity", Erice, December 1992 and private communication; "Four-dimensional gravitational backgrounds based on $N=4, \hat{c}=4$ superconformal systems" CERNTH 6799/93, hep-th/9304102.

[6] W.Lerche, C.Vafa and N.P.Warner, Nucl. Phys. B234 (1989) 427.

[7] C.Kounnas, M.Porrati and B.Rostand, Phys. Lett. B258 (1991) 61.

[8] R.D'Auria and T.Regge Nucl. Phys. B195 (198?) 308.

[9] E.Ivanov and S.Krivonos, J. Phys. A: Math. Gen. 17 (1984) L671; E.Ivanov, S.Krivonos and V.Leviant,Int. Jour. Mod. Phys. A7 (1992) 287.

[10] T.Eguchi and P.G.O.Freund, Phys. Rev. Lett. 37 (1976) 1251; S.W.Hawking, Phys. Lett. 60A (1977) 81.

[11] G.W.Gibbons, S.Hawking, Commun. Math. Phys. 66 (1979) 291. 
[12] T.Eguchi, A.J.Hanson, Phys. Lett. 74B (1978) 249, Ann. Phys. 120 (1979) 82.

[13] S.W.Hawking, C.N.Pope, Nucl. Phys. B146 (1978) 381.

[14] N.J.Hitchin, Math. Proc. Camb. Phyl. Soc. 85 (1979) 465.

[15] P.B.Kronheimer, J. Differ. Geometry 29 (1989) 665.

[16] P.B.Kronheimer, J. Differ. Geometry 29 (1989) 685.

[17] L.Dixon, J.Harvey, C.Vafa and E.Witten, Nucl. Phys. B261 (1985) 620, B274 (1986) 285.

[18] L.Dixon, Lectures in Superstrings, Unified Theories and Cosmology 1987 G.Furlan, J.C.Pati, D.W.Sciama and Q.Shafi eds., World Scientific, Singapore, 1988.

[19] T.Eguchi, P.B.Gilkey, A.J.Hanson, Phys. Rep. 66 (1980) 213.

[20] P.Griffits and J.Harris, Priciples of Algebraic Geometry, Wiley-Interscience, NewYork, 1978.

[21] G.W.Gibbons, S.W.Hawking, Phys. Lett. 78B (1978) 430.

[22] F.Klein, Lectures on the Icosahedron and the solution of an equation of fifth degree, Dover, New-York, 1913 (English translation).

[23] Plato, Timaeus $\mu \epsilon v \sigma$.

[24] F.D.Murnagham, The Theory of Group Representations, Dover, New-York, 1963

[25] J.Mc Kay in Proc. Symp. Pure Math., Am. Math. Soc. v37 (1980) 183.

[26] M.Artin, Amer. J. Math. 88 (1966) 129; V.I.Arnold, S.M.Gusein-Zade and A.N.Varčenko, Singularities of Differentiable Maps, Birkhäuser, Boston 1988; E.Brieskorn in Actes Congrés Intern. Math. Ann. 1970, t.2 279; P.Slodowy, Simple Singularities and Simple Algebraic Groups Lecture Notes in Mathematics v.815 (1980) Springer-Verlag.

[27] N.J.Hitchin, A.Karlhede, U.Lindström, M.Roček, Commun. Math. Phys. 108 (1987) 535 .

[28] U.Lindström, M.Roček, Nucl. Phys. B222 (1983) 285

[29] L.Dixon, D.Friedan, E.Martinec, S.Shenker, Nucl. Phys. B282 (1987) 13.

[30] T.Eguchi and A.Taormina, Phys. Lett. B196 (1987) 75, B200 (1988) 315, B210 (1988) 125.

[31] R,Dijkgraf, E.Verlinde and H.Verlinde, Nucl. Phys. B352 (1991) 59. 
[32] T.Eguchi, H.Ooguri, A.Taormina and S.K.Yang Nucl. Phys. B315 (1989) 193.

[33] P.Ginsparg, Les Houches lectures 1988, ed. by E.Brezin and J.Zinn-Justin, Elsevier Science Publishers B.V. (1989).

[34] I.Antoniadis, C.Bachas, J.Ellis and D.Nanopoulos, Phys. Lett. B211 (1988) 393, Nucl. Phys B328 (1989) 117.

[35] A.Cappelli, C.Itzykson and J.B.Zuber, Nucl. Phys. B280 (1987) 445, Commun. Math. Phys. 113 (1987) 1; A.Kato, Mod. Phys. Lett. A2 (1987) 585. 